\title{
Protecting the Conformal Symmetry via Bulk Renormalization on Anti deSitter Space*
}

\author{
Michael Düitsch ${ }^{1,2}$, Karl-Henning Rehren ${ }^{1,2}$ \\ ${ }^{1}$ Institut für Theoretische Physik, Universität Göttingen, Friedrich-Hund-Platz 1, 37077 Göttingen, Germany. \\ E-mail: michael.duetsch@theorie.physik.uni-goettingen.de; rehren@ theorie.physik.uni-goettingen.de \\ 2 Courant Research Centre "Higher Order Structures in Mathematics", Universität Göttingen, Bunsenstr. 3-5, \\ 37073 Göttingen, Germany
}

Received: 26 March 2010 / Accepted: 20 February 2011

Published online: 24 August 2011 - (C) The Author(s) 2011. This article is published with open access at Springerlink.com

\section{Dedicated to Raymond Stora on the occasion of his 80th birthday}

\begin{abstract}
The problem of perturbative breakdown of conformal symmetry can be avoided, if a conformally covariant quantum field $\varphi$ on $d$-dimensional Minkowski spacetime is viewed as the boundary limit of a quantum field $\phi$ on $d+1$-dimensional AntideSitter spacetime (AdS). We study the boundary limit in renormalized perturbation theory with polynomial interactions in $\mathrm{AdS}$, and point out the differences as compared to renormalization directly on the boundary. In particular, provided the limit exists, there is no conformal anomaly. We compute explicitly the one-loop "fish diagram" on $\mathrm{AdS}_{4}$ by differential renormalization, and calculate the anomalous dimension of the composite boundary field $\varphi^{2}$ with bulk interaction $\kappa \phi^{4}$.
\end{abstract}

\section{Contents}

1. Introduction . . . . . . . . . . . . . . . 315

2. The General Strategy . . . . . . . . . . . . . . . . . . . . . . . . . . . . . . . . . . . .

3. Case Studies I: The Interacting Boundary Field $\varphi_{\kappa \phi^{k}} \ldots$. . . . . . . . . . . 329

4. Case Studies II: The Interacting Composite Field $\left(\varphi^{2}\right)_{\kappa \phi^{k}} \ldots \ldots$. . . . . . . . . . 336

5. Conclusion . . . . . . . . . . . . . . . . . 343

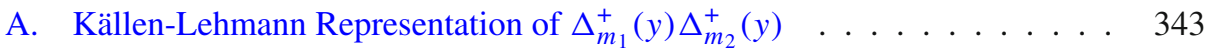

B. The Origin of the Logarithmic Boundary Terms . . . . . . . . . . . . . . 344

C. Details of the Renormalization of the Massless Fish Diagram on AdS . . . 347

D. Integrals for the Boundary Limit . . . . . . . . . . . . . . . . . . . 349

\section{Introduction}

When a scale invariant free field is perturbed by an interaction, the scaling symmetry is in general broken. In the case of the free massless scalar field in 4-dimensional Minkowski

\footnotetext{
* Supported in part by the German Research Foundation (Deutsche Forschungsgemeinschaft (DFG)) through the Institutional Strategy of the University of Göttingen, and DFG Grant RE 1208/2-1.
} 
space, this "conformal anomaly" is well known: the renormalization of loop diagrams requires the introduction of a scale parameter which breaks scale invariance. Using the non-uniqueness of renormalization, the best one can reach is "almost homogeneous scaling", i.e. the breaking terms for the scaling $x \mapsto \lambda x$ are proportional to some power of $\log \lambda$. (For a systematic treatment in the framework of causal perturbation theory see $[9,17,18]$.)

In this paper, we want to address the analogous issue for scale invariant generalized free fields (free fields with non-canonical scaling dimension, see (2.9) below). Such fields naturally arise as boundary limits of Klein-Gordon fields on AdS [3,4,29]. The basic question is:

- Is it possible to construct scale invariant interacting fields (admitting for anomalous dimensions)

$$
\left(\varphi^{l}\right)_{\kappa L}(x)=: \varphi^{l}(x):+\mathcal{O}(\kappa)
$$

as perturbative expansions around Wick powers : $\varphi^{l}(x)$ : of scale invariant generalized free fields $\varphi[11]$ ?

( $L$ denotes the interaction density and $\kappa$ the coupling constant.)

Perturbation theory around a generalized free field (in Minkowski space) suffers from a huge arbitrariness which is due to renormalization, as we point out in Sect. 2. On the other hand, the requirement of scale invariance is very restrictive. In important cases (which we do not want to exclude) it cannot be fulfilled even for tree diagrams (Sect. 3.4). Namely, the propagator needs a nontrivial renormalization if the scaling dimension $\Delta$ is $\geq 2$ in four dimensions ( $\frac{d}{2}$ in $d$ dimensions), and for integer $\Delta$ a breaking of scale invariance cannot be avoided.

We propose here a method to circumvent these difficulties and construct perturbatively interacting fields with unbroken conformal symmetry, by taking advantage of the AdS-CFT correspondence. Viewing a conformally covariant field on Minkowski space-time as a boundary limit of an AdS covariant field on Anti-deSitter space-time $[3,4,10,29]$, an AdS invariant renormalization in the bulk guarantees an anomaly free conformal symmetry of the boundary field, provided the boundary limit exists. In this way, the AdS-CFT correspondence turns out to be a useful tool also when one is only interested in CFT in Minkowski spacetime.

In $[3,4]$ and [11] it was shown that the boundary limit $z \searrow 0^{1}$ of the scalar KleinGordon field $\phi(z, x)$ of mass $M$ on $(d+1)$-dimensional AdS is a generalized free field $\varphi(x)$ with scaling dimension

$$
\Delta=\Delta_{+}=\frac{d}{2}+v, \quad\left(v=\sqrt{\frac{d^{2}}{4}+M^{2}}\right),
$$

see Sect. 2. The corresponding boundary limit of the free Wick powers $W(z, x)=$ $: \phi^{l}(z, x)$ : yields fields $w(x)=: \varphi^{l}(x)$ : which have scaling dimensions $l \Delta$. Notice that in the Witten model [29] of Maldacena's conjectured AdS-CFT correspondence [22], one studies instead the "dual" field with boundary conditions corresponding to $\Delta_{-}=\frac{d}{2}-v$, which is coupled to the sources in a "dual" way. However, it was shown

${ }^{1}$ We use Poincaré coordinates $X \equiv\left(z, x^{\mu}\right) \in \mathbb{R}_{+} \times \mathbb{R}^{d}$ of $\operatorname{AdS}_{d+1}$ such that $\xi=z^{-1}\left(x^{\mu}, \frac{1}{2}\left(z^{2}-x^{2}-1\right)\right.$, $\left.\frac{1}{2}\left(z^{2}-x^{2}+1\right)\right)$ lies on the hyperboloid $\xi \cdot \xi=1$ w.r.t. to the metric of signature $(+,-\ldots-,+)$ in the ambient space $\mathbb{R}^{d+2}$. The AdS metric is the induced one: $d s^{2}=z^{-2}\left(d x_{\mu} d x^{\mu}-d z^{2}\right)$, see e.g. [3,4]. 
in [10] that the dual coupling modifies the relevant bulk propagator by a correction term in such a way, that the full propagator becomes that of the above Klein-Gordon field, and the unrenormalized perturbative expansion of the dually coupled boundary field is formally equivalent to the boundary limit of the bulk field $\phi(z, x)$ with the same interaction. (The same nontrivial features, that are of representation theoretic nature, were established for the propagators of symmetric tensor fields of any rank [26].)

Regarding the generalized free field as a limit of a canonical free field on AdS, the task is to extend this relation to the renormalized interacting fields. Hence, we first construct the interacting AdS fields

$$
W_{\kappa \mathcal{L}}(z, x)=: \phi^{l}(z, x):+\mathcal{O}(\kappa)
$$

for polynomial interactions $\mathcal{L}=\phi^{k}$ in Sect. 3 and Sect. 4, using standard renormalization methods of causal perturbation theory (reviewed in Sect. 2.2 and 2.3). At this stage, the non-uniqueness of the renormalization can be classified by the usual short distance power counting $[6,17,18]$, and the propagator is unique and AdS-invariant, hence the AdS symmetry is fully preserved.

Then, the essential step is to investigate the existence of a boundary limit

$$
w_{\kappa \mathcal{L}}(x)=\lim _{z \searrow 0} z^{-\Delta_{\kappa \mathcal{L}}^{W}} \cdot W_{\kappa \mathcal{L}}(X)
$$

in the renormalized theory. Here, we admit for anomalous dimensions, i.e., $\Delta_{\kappa \mathcal{L}}^{W}=$ $l \Delta+\mathcal{O}(\kappa)$. If this limit exists, we prove that it inherits the AdS symmetry of the bulk as an exact (unbroken) conformal symmetry (Sect. 2.4).

Our main result is that the boundary limit does exist, for typical polynomial interactions, for the interacting field (Sect. 3) and for composite fields (Sect. 4), due to nontrivial cancellations within the renormalized one-loop distributions taking place in the limit. Although the actual computations are "hidden" in Apps. C and D, these cancellations constitute the essential mechanism to allow the passage to the boundary.

In order to establish this result, along the way we develop a "universal" formula (Lemma B.1 in App. B) that controls the asymptotic behaviour near the boundary of a large class of typical interactions and diagrams.

Thus, the above posed question gets an affirmative answer for those interactions $L[\varphi(x)]$ of the conformal field which are "induced" by the corresponding polynomial AdS interaction $\mathcal{L}[\phi(X)]$ (as indicated by retaining the subscript $\kappa \mathcal{L}$ in (1.4) also for the boundary field). This means [11] that

$$
\kappa \int d^{d} x L[\varphi(x)]=\kappa \int d z d^{d} x \sqrt{-g} \mathcal{L}[\phi(z, x)],
$$

hence the CFT interaction density

$$
L[\varphi(x)]=\int d z \sqrt{-g} \mathcal{L}[\phi(z, x)]=\int \frac{d z}{z^{d+1}} \mathcal{L}\left[\varphi_{h_{z}}(x)\right]
$$

arises as the $z$-integral over $\mathcal{L}\left[\varphi_{h_{z}}(x)\right]$, where $\varphi_{h_{z}}(x)$ is the AdS field $\phi(z, x)$ re-expressed as a family of boundary generalized free fields belonging to the Borchers class of $\varphi$ ([11], see Sect. 2.1). We point out that, due to the integration in (1.6), the interaction vertices "remain in the bulk". In this sense, the situation is converse to Rühl's reconstruction [27] of an AdS field from an interacting conformal field where the AdS interaction is 
restricted to the boundary (namely, the AdS field in [27] satisfies the free field equation in the bulk).

It is an essential aspect of our approach that, while the general principles of renormalization are the same, the detailed implementation of the rules differ in the bulk and on the boundary. In order to exhibit the methodic difference which allows the renormalization in the bulk to preserve the symmetry that is necessarily broken by renormalization on the boundary, we compare both approaches in Sect. 2.5 with a flat space toy model, where this difference is much more transparent.

\section{The General Strategy}

2.1. Free fields. Let us recall [11] how the Klein-Gordon field on $(d+1)$-dimensional Anti-deSitter space and generalized free fields on $d$-dimensional Minkowski space can be represented in terms of the same creation and annihilation operators, and hence as field operators on the same Hilbert space.

The free Klein-Gordon field $\phi$ of mass $M$ on AdS can be expressed as

$$
\phi(z, x)=\frac{1}{\sqrt{2}} z^{\frac{d}{2}} \int_{0}^{\infty} d m^{2} J_{v}(m z) \varphi_{m}(x),
$$

where $\varphi_{m}$ is a massive free boundary field given by

$$
\varphi_{m}(x) \equiv \int_{k_{0} \geq 0} d^{d} k \delta\left(k^{2}-m^{2}\right)\left[a(k) e^{-i k x}+a^{+}(k) e^{i k x}\right] .
$$

The parameter $v>-1$ is related to the mass by $M^{2}=v^{2}-\frac{d^{2}}{4}$. The functions $z^{d / 2} J_{v}\left(\sqrt{k^{2}} z\right) \exp \pm i k x$ are the plane-wave solutions to the Klein-Gordon equation on $\mathrm{AdS}$, where the Laplacian is

$$
\square_{X}=-z^{1+d} \partial_{z} z^{1-d} \partial_{z}+z^{2} \square_{x}
$$

and $a(k), a^{+}(k)\left(k \in \mathbb{R}^{d}\right)$ are creation and annihilation operators normalized as

$$
\left[a(k), a^{+}\left(k^{\prime}\right)\right]=(2 \pi)^{-(d-1)} \delta^{d}\left(k-k^{\prime}\right), \quad[a, a]=0=\left[a^{+}, a^{+}\right],
$$

in the Fock space $\mathcal{H}$ over the continuous mass 1-particle space $\mathcal{H}_{1}=L^{2}\left(V_{+}, d^{d} k\right)$.

In this Hilbert space, the fields

$$
\varphi_{h}(x) \equiv \int_{V_{+}} d^{d} k h\left(k^{2}\right)\left[a(k) e^{-i k x}+a^{+}(k) e^{i k x}\right]
$$

(with $h$ any sufficiently smooth polynomially bounded real function on $\mathbb{R}_{+}$) are local and Poincaré covariant generalized free scalar fields in $d$-dimensional Minkowski space with Källen-Lehmann measure $d \mu\left(m^{2}\right)=h\left(m^{2}\right)^{2} d m^{2}$. Thus, $\phi$ may be written as

$$
\phi(z, x)=\varphi_{h_{z}}(x) \text { with } h_{z}\left(m^{2}\right) \equiv \frac{1}{\sqrt{2}} z^{\frac{d}{2}} J_{v}(z m) .
$$

Taking the boundary limit, we get $[3,4,11]$ :

$$
\lim _{z \searrow 0} z^{-\Delta} \phi(z, x)=\varphi(x)
$$


with $^{2}$

$$
\varphi(x)=C_{v} \int_{V_{+}} d^{d} k\left(k^{2}\right)^{\frac{v}{2}}\left[a(k) e^{-i k x}+a^{+}(k) e^{i k x}\right], \quad \Delta \equiv v+\frac{d}{2},
$$

i.e., $\varphi=\varphi_{h}$ with $h\left(m^{2}\right)=C_{v} m^{v}, C_{v} \equiv \frac{2^{-v-\frac{1}{2}}}{\Gamma(v+1)}$. Its Källen-Lehmann measure being a homogeneous function of the mass:

$$
d \mu\left(m^{2}\right)=C_{v}^{2} m^{2 v} d m^{2},
$$

the boundary field $\varphi$ is scale invariant:

$$
U(\lambda) \varphi(x) U(\lambda)^{*}=\lambda^{\Delta} \varphi(\lambda x),
$$

and in fact transforms like a conformal scalar field under the representation of the AdS symmetry group on the Fock space of the AdS Klein-Gordon field $\phi$.

The boundary limit (2.7) can obviously be generalized to arbitrary Wick polynomials $W=: \prod_{j=1}^{l} \partial_{x}^{a_{j}} \phi:$

$$
w(x)=\lim _{z \searrow 0} z^{-l \Delta} W(z, x)=: \prod_{j=1}^{l} \partial^{a_{j}} \varphi(x):,
$$

which have scaling dimension $D^{W}=l \Delta+\sum_{j}\left|a_{j}\right|$ (where $a_{j} \in\left(\mathbb{N}_{0}\right)^{d}$ is a multi-index).

2.2. Causal perturbation theory. The aim of this paper is to investigate causal perturbation theory [13] around the generalized free field (2.8) (and its Wick polynomials (2.11)). Causal perturbation theory proceeds $[6,9,13]$ by defining, for each Wick polynomial $W$ of free fields $\phi$, the interacting field $W_{g \mathcal{L}}$ as formal expansion in Wick products of the free field $\phi$ with distributional coefficients. This expansion is obtained as the exponential series of retarded products of $W$ with the interaction $g \mathcal{L}$, where the retarded products are operator-valued distributions. They are determined recursively (by the postulated causal properties of the interacting fields) at non-coinciding points only; the renormalization of the perturbative expansion consists in the extension of these distributions to coinciding points. "Renormalization conditions" (covariance, Ward identities, ...) serve to reduce the arbitrariness in the extension, and the main problem is to decide whether all desirable renormalization conditions can be fulfilled at the same time, with a finite number of free parameters remaining.

This program is performed with the interaction being cut off in space and time by means of a space-time dependent coupling constant $g(x)$. It then remains to control the adiabatic limit of removing the cutoff, $g(x) \rightarrow \kappa$. This limit is in general plagued by infrared problems; it is, however, possible to define the algebraic adiabatic limit [6], i.e., the local field algebras $\mathcal{F}_{\kappa \mathcal{L}}(K)$ in arbitrary bounded space-time regions $K$, without infrared problems as long as the construction of the interacting vacuum state is postponed.

Causal perturbation theory around a generalized free field is, however, problematic for the following reason. To construct the general solution for the perturbative $S$-matrix

${ }^{2}$ It should not lead to confusion that the present field $\varphi$ was denoted $\varphi^{(\Delta)}$ in [11], whereas $\varphi_{h}$ with $h\left(m^{2}\right)=1$ was denoted $\varphi$. 
one has to use the Wick expansion formula for time-ordered or retarded products (also called the "causal Wick expansion") [6,9,13]. For simplicity, let us discuss here the ordinary Wick expansion formula, which for mass shell free fields is

$$
\begin{aligned}
& : \varphi_{m}^{k_{1}}\left(x_{1}\right): \ldots: \varphi_{m}^{k_{n}}\left(x_{n}\right): \\
& \quad=\sum_{r_{1}, \ldots, r_{n}} \prod_{i=1}^{n}\left(\begin{array}{c}
k_{i} \\
r_{i}
\end{array}\right)\left(\Omega,: \varphi_{m}^{k_{1}-r_{1}}\left(x_{1}\right): \ldots: \varphi_{m}^{k_{n}-r_{n}}\left(x_{n}\right): \Omega\right) \cdot: \varphi_{m}^{r_{1}}\left(x_{1}\right) \ldots \varphi_{m}^{r_{n}}\left(x_{n}\right): .
\end{aligned}
$$

For generalized free fields, the Lagrangian can be any field relatively local w.r.t. the generalized free field, i.e., any element of its Borchers class. The Borchers class contains at least the "generalized Wick polynomials" [11]

$$
\begin{aligned}
& \left(: \varphi^{l}:\right)_{h}(x) \\
& \quad=\int_{V_{+}} d^{d} k_{1} \ldots \int_{V_{+}} d^{d} k_{l} h\left(k_{1}^{2}, \ldots, k_{l}^{2}\right) \cdot:\left[a\left(k_{1}\right) e^{-i k_{1} x}+\text { h.c. }\right] \ldots\left[a\left(k_{l}\right) e^{-i k_{l} x}+\text { h.c. }\right]:,
\end{aligned}
$$

where $h:\left(\mathbb{R}_{+}\right)^{l} \rightarrow \mathbb{C}$ is any symmetric and sufficiently regular function. Let us choose a Lagrangian $L(y)=\left(: \varphi^{4}:\right)_{H}(y)$ with an arbitrary function $H\left(k_{1}^{2}, \ldots, k_{4}^{2}\right)$. It is then easy to see, that the Wick expansion of, say, $\varphi_{h}(x)$ with $L(y)$ does not factorize as in (2.12), but rather contains terms of the form

$$
\begin{aligned}
& \int_{V_{+}} d^{d} k_{1} \ldots d^{d} k_{3} h\left(x-y ; k_{1}^{2}, k_{2}^{2}, k_{3}^{2}\right) \\
& \quad \times:\left[a\left(k_{1}\right) e^{-i k_{1} y}+\text { h.c. }\right] \ldots\left[a\left(k_{3}\right) e^{-i k_{3} y}+\text { h.c. }\right]:,
\end{aligned}
$$

where

$$
h\left(x-y ; k_{1}^{2}, k_{2}^{2}, k_{3}^{2}\right)=\int_{V_{+}} d q e^{-i q(x-y)} h\left(q^{2}\right) H\left(q^{2}, k_{1}^{2}, k_{2}^{2}, k_{3}^{2}\right) .
$$

Because the dependence of this function on $x-y$ and on $k_{i}^{2}$ is entangled in a nontrivial manner, the numerical distribution cannot be separated from the operator-valued distribution as in (2.12) (unless $H$ happens to be a factorizing function). Interpreting (2.14) as an operator product expansion, reveals a characteristic feature of the theory of generalized free fields: performing first the $k$-integrations, the subsequent $q$-integration may be interpreted as a "continuous sum" over generalized Wick products. More importantly, however, the failure of separation as in (2.12) would require more refined methods to establish the existence of a renormalization, than the standard methods of causal perturbation theory, which proceeds by renormalizing only the numerical distributions (see below).

Let us contrast the general case to the case when the interaction is induced by a local interaction on AdS [11] as described in the introduction, i.e., when the conformal field $\varphi$ arises as the boundary limit of a canonical AdS field $\phi$ with interaction $\kappa \mathcal{L}$. The Lagrangian $L$ given by (1.6) with, say, $\mathcal{L}=\phi^{4}$ on $\operatorname{AdS}$ is $L=\left(: \varphi^{4}:\right)_{H}$ with

$$
H\left(k_{1}^{2}, \ldots, k_{4}^{2}\right)=\int d z z^{-d-1} \prod h_{z}\left(k_{i}^{2}\right)
$$


i.e., $H$ is a $z$-integral over factorizing functions; one can therefore reorganize the continuous OPE as a $z$-integral over Wick products of the distinguished fields $\varphi_{h_{z}}(x)$ as in (2.6), rather than generalized Wick products as in (2.13). This fact seems to reduce the renormalization ambiguity drastically, since the freedom is only in the choice of suitable weight functions in $z$. Whether a conformally covariant renormalization of the OPE of perturbed boundary fields is possible, would require a nontrivial analysis.

This is the reason why we propose to work instead with the "bulk approach" mentioned before, using the correspondence (2.7) and (2.11); i.e., we first construct the perturbative interacting fields on $(d+1)$-dimensional Anti-deSitter space $[6,17,18]$, and then study their boundary limit. We shall see that conformal covariance can be maintained on the boundary because AdS covariance can be maintained in the bulk. The issue therefore has been shifted to the existence of the limit. It will be illustrated in Sect. 2.5, why this indirect approach gives different results than the direct approach perturbing generalized free fields on the boundary.

In [6] and $[17,18]$ perturbative interacting fields have been constructed on an arbitrary globally hyperbolic curved spacetime $\mathcal{M}$ for localized interactions $G(x) \mathcal{L}(x)$, i.e., the interaction $\mathcal{L}$ is switched on by $G \in \mathcal{D}(M)$. The Anti-deSitter spacetime is not itself globally hyperbolic, but its covering is conformally equivalent to a $\mathbb{Z}_{2}$ quotient of a globally hyperbolic space-time [2]. In this way, the lack of global hyperbolicity can be circumvented in terms of boundary conditions "at infinity" $(z=0)$.

If one wants to take the boundary limit, one obviously must not cut off the interaction on the boundary of AdS, hence we must perform a "partial adiabatic limit" which puts the switching function $G(z, x)$ to be 1 for $x \in K$ (a compact region $\subset \mathbb{M}_{d}$ ) and $z=0$. It can be easily seen that the conclusion of [6], i.e., the independence of the algebraic adiabatic limit on the details of the switching function outside the compact region of interest, holds also true for the partial adiabatic limit. We may therefore assume that the switching function factorizes as

$$
G(z, x)=\kappa \gamma(z) g(x), \text { where }\left.G\right|_{[0, a] \times K} \equiv \kappa=\text { constant }
$$

with $\left.g\right|_{K} \equiv 1$ and $\left.\gamma\right|_{[0, a]} \equiv 1$ for some $a>0$. In addition $g$ and $\gamma$ are smooth, supp $g$ is compact and the support of $\gamma(z)$ is bounded for $z \rightarrow \infty$. Since the support of such functions $G$ is not compact in AdS, there may in principle be IR problems associated with the partial adiabatic limit; but our explicit calculations in Sect. 3 show that these do not appear in the relevant examples. The (partial) algebraic adiabatic limit does not depend on the details of the functions $g$ and $\gamma$, provided $a$ is sufficiently large.

In practice, we proceed as follows: Given a Wick monomial $w$ in the generalized free field $\varphi$ and its derivatives, we first replace $\varphi(x)$ by the AdS field $\phi(z, x)$ (whose boundary limit is $\varphi(x))$, and construct the interacting $\operatorname{AdS}$ field $W_{\kappa \mathcal{L}}(z, x)$ associated with the corresponding Wick monomial $W$ in $\phi$ and its derivatives. Then we define the interacting field $w_{\kappa \mathcal{L}}(x)$ in $\mathbb{M}_{d}$ as boundary limit of the interacting field $W_{\kappa \mathcal{L}}(z, x)$ on AdS, provided this limit exists:

$$
w_{\kappa \mathcal{L}}(x)=\lim _{z \searrow 0} z^{-\Delta_{\kappa \mathcal{L}}^{W} W_{\kappa} \mathcal{L}}(z, x)
$$

where

$$
\Delta_{\kappa \mathcal{L}}^{W}=l \Delta+\sum_{n=1}^{\infty} \kappa^{n}\left(\Delta_{\mathcal{L}}^{W}\right)^{(n)}
$$


The deformation $l \Delta \mapsto \Delta_{\kappa \mathcal{L}}^{W}$ (i.e., the sequence of coefficients $\left.\left(\Delta_{\mathcal{L}}^{W}\right)^{(n)} \in \mathbb{C}, n \geq 1\right)$ is determined by the requirement that the limit (2.18) exists.

Remark. In ordinary perturbative QFT the anomalous dimension is the deviation of the scaling dimension of an (interacting) quantum field $A_{\kappa} \mathcal{L}$ from the scaling dimension of the corresponding (interacting) classical field. For generalized free fields there is no obvious classical counterpart. Instead, we call "anomalous dimension of $w_{\kappa} \mathcal{L}$ " the deformation of the scaling dimension due to the interaction. In contrast to ordinary perturbative QFT, it does not come from the breaking of scale invariance in the renormalization of loop diagrams (we maintain the AdS-symmetry in the renormalization). Instead its appearance is enforced by the existence of the boundary limit.

In causal perturbation theory on $\mathrm{AdS}, W_{\kappa \mathcal{L}}$ is given by $[6,17,18]$

$$
\begin{aligned}
W_{\kappa \mathcal{L}}(X)= & \sum_{n=0}^{\infty} \frac{\kappa^{n}}{n !}\left(\prod_{r=1}^{n} \int_{0}^{\infty} \frac{d z_{r}}{z_{r}^{d+1}} \gamma\left(z_{r}\right) \int d^{d} x_{r} g\left(x_{r}\right)\right) \\
& \cdot R_{n, 1}\left(\mathcal{L}\left(X_{1}\right), \ldots, \mathcal{L}\left(X_{n}\right) ; W(X)\right),
\end{aligned}
$$

where $X \equiv(z, x)$ and $X_{j} \equiv\left(z_{j}, x_{j}\right)$. The unrenormalized retarded products $R_{n, 1}$ are determined as distributions at non-coinciding points $X_{i} \neq X_{j} \neq X$.

The result is $[8,20]$

$$
\begin{aligned}
& R_{n, 1}\left(\mathcal{L}\left(X_{1}\right), \ldots \mathcal{L}\left(X_{n}\right) ; W(X)\right)=(-i)^{n} n ! \\
& \quad \times \mathcal{S}\left[\theta\left(x^{0}>x_{n}^{0}>x_{n-1}^{0}>\cdots>x_{1}^{0}\right) \cdot\left[\mathcal{L}\left(X_{1}\right),\left[\mathcal{L}\left(X_{2}\right) \ldots\left[\mathcal{L}\left(X_{n}\right), W(X)\right] \ldots\right]\right]\right],
\end{aligned}
$$

where $\mathcal{S}$ means symmetrization in $X_{1}, \ldots, X_{n}$.

Now let $W=: \prod_{j=1}^{l} \partial_{x}^{a_{j}} \phi:$ and $\mathcal{L}=: \phi^{k}$ : . Then, using (2.6) and (2.1), the retarded product (2.21) may be rewritten as

$$
\begin{aligned}
R_{n, 1}\left(: \varphi_{h_{z_{1}}}^{k}\left(x_{1}\right):, \ldots,: \varphi_{h_{z n}}^{k}\left(x_{n}\right): ;: \prod_{j=1}^{l} \partial_{x}^{a_{j}} \varphi_{h_{z}}(x):\right) \\
=(-i)^{n} n ! \mathcal{S}\left[\theta\left(x^{0}>x_{n}^{0}>x_{n-1}^{0}>\cdots>x_{1}^{0}\right)\right. \\
\quad \cdot \frac{\left(z_{1}^{k} \ldots z_{n}^{k} z^{l}\right)^{d / 2}}{2^{(l+n k) / 2}} \int \prod_{r=1}^{n} \prod_{s=1}^{k} d m_{r s}^{2} J_{v}\left(m_{r s} z_{r}\right) \int \prod_{j=1}^{l} d m_{j}^{2} J_{v}\left(m_{j} z\right) \\
\left.\cdot\left[: \prod_{s} \varphi_{m_{1 s}}\left(x_{1}\right):, \ldots\left[: \prod_{s} \varphi_{m_{n s}}\left(x_{n}\right):,: \prod_{j} \partial^{a_{j}} \varphi_{m_{j}}(x):\right] \ldots\right]\right] .
\end{aligned}
$$

We emphasize that writing (2.21) as in the left-hand side of (2.22) is misleading: It is not a retarded product in Minkowski space, but in AdS, defined with respect to the causal structure in AdS. In particular, the problem with the causal Wick expansion for generalized free fields mentioned before, is absent, and its correct definition is the righthand side of (2.22). Moreover, renormalization is needed for coinciding AdS points $X_{i}=X$ only, and not on the whole submanifold $x_{i}=x$, as will be discussed in the next subsection. 


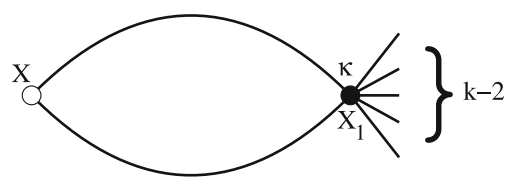

Fig. 1. The "fish" diagram arising in first order perturbation theory for the interacting field $\left(\phi^{2}\right)_{\kappa \mathcal{L}}(X)$ with interaction $\mathcal{L}=\phi^{k}$. The diagram symbolizes the distribution $r_{\text {fish }}\left(X_{1} ; X\right) \equiv$ $\left(\Omega, R_{1,1}\left(: \phi^{2}\left(X_{1}\right):,: \phi^{2}(X):\right) \Omega\right.$ ) or the corresponding unrenormalized expression $r_{\text {fish }}^{\circ}$ (given by (2.44) or (4.2) resp., appearing in the second line of (2.24))

In the sequel, we shall be mainly concerned with special cases of the type

$$
R_{1,1}\left(: \phi^{k}\left(X_{1}\right): ; \phi(X)\right)=k i \Delta\left(X ; X_{1}\right) \theta\left(x^{0}-x_{1}^{0}\right) \cdot: \phi\left(X_{1}\right)^{k-1}:
$$

and

$$
\begin{aligned}
& R_{1,1}\left(: \phi^{k}\left(X_{1}\right): ;: \phi^{2}(X):\right)=2 k i \Delta\left(X ; X_{1}\right) \theta\left(x^{0}-x_{1}^{0}\right) \cdot: \phi\left(X_{1}\right)^{k-1} \phi(X): \\
& \quad+k(k-1) i\left(\Delta^{+}\left(X ; X_{1}\right)^{2}-\Delta^{+}\left(X_{1} ; X\right)^{2}\right) \theta\left(x^{0}-x_{1}^{0}\right) \cdot: \phi\left(X_{1}\right)^{k-2}:,
\end{aligned}
$$

where $\Delta^{+}\left(X ; X_{1}\right)=\left(\Omega, \phi(X) \phi\left(X_{1}\right) \Omega\right)$ is the scalar 2-point function, and $\Delta\left(X ; X_{1}\right)=$ $\left(\Omega,\left[\phi(X), \phi\left(X_{1}\right)\right] \Omega\right)$ the commutator function.

2.3. The problem of renormalization. The expressions (2.21)-(2.24) are not defined as distributions at coinciding points, due to the time-ordering $\theta$ functions. The problem of renormalization is thus the extension of the retarded products to distributions $R_{n, 1}(\ldots)$ on $\left(\mathbb{R}_{+} \times \mathbb{R}^{d}\right)^{n+1}$. By the recursive construction principle underlying causal perturbation theory, once this has been achieved for $R_{l, 1}(l<n)$, then $R_{n, 1}$ is already determined everywhere outside the total diagonal

$$
\Delta_{n+1} \equiv\left\{\left(X_{1}, \ldots, X_{n} ; X\right) \mid X_{j}=X \forall j=1, \ldots, n\right\}
$$

Renormalization at $n^{\text {th }}$ order is thus reduced to the extension of the distributions $R_{n, 1}$ from $\left(\mathbb{R}_{+} \times \mathbb{R}^{d}\right)^{n+1} \backslash \Delta_{n+1}$ to $\left(\mathbb{R}_{+} \times \mathbb{R}^{d}\right)^{n+1}$.

Applying the recursion as indicated, gives rise to a diagrammatic expansion of $R_{n, 1}$ in terms of Wick products with propagators and numerical distributions $r_{m, 1}^{\circ}(\ldots)(m \leq n)$ as coefficients, as in (2.24). The latter are the vacuum expectation values of operatorvalued distributions (with field arguments of possibly lower order). E.g., for $W=: \phi^{2}$ : and $\mathcal{L}=: \phi^{k}:$, there arises the "fish diagram" (Fig. 1) as the coefficient of : $\phi^{k-2}\left(X_{1}\right)$ : to first order in $\kappa$.

Renormalization is done in terms of the numerical distributions $r_{m, 1}^{\circ}$, by extending them to distributions $r_{m, 1}$ on $\left(\mathbb{R}_{+} \times \mathbb{R}^{d}\right)^{m+1}$. (For an example in flat space, see Sect. 2.5.) We shall see, however, that the $z \searrow 0$ behaviour of the renormalized operator-valued distributions $R_{n, 1}$ on AdS is in general not the same as that of the numerical distributions $r_{m, 1}$; thus the existence of the limit has to be studied for the operator-valued distribution $R_{n, 1}$. 
For a rigorous and complete definition of the retarded products $R_{n, 1}$ we refer to the renormalization axioms given in $[9],{ }^{3}$ with appropriate modifications due to the curvature of AdS $[6,17,18]$. In particular, the renormalization should not increase the scaling degree of a distribution [6], which controls the "strength of the UV singularity": The scaling degree is defined in flat space by

$$
\operatorname{sd}_{Y}(f(\cdot ; X))=\inf \left\{\delta \in \mathbb{R} \mid \lim _{\lambda \searrow 0} \lambda^{\delta} f(X+\lambda Y ; X)=0\right\}
$$

where the limit is meant as a distribution in $Y \in \mathbb{R}^{d+1}$; in curved spacetime, $Y$ is taken in the tangent space and the argument $X+\lambda Y$ has to be replaced by the geodesic exponential $\exp _{X}(\lambda Y)$.

Moreover, the renormalization conditions of translation invariance and $\mathcal{L}_{+}^{\uparrow}$-covariance are replaced by AdS-invariance (group $S O(2, d)$ ). The expression $(2.21)$ is obviously AdS-invariant, so the problem consists in the preservation of this symmetry upon renormalization.

Since we construct the interacting field on AdS, $R_{n, 1}\left(\mathcal{L}\left(X_{1}\right), \ldots, \mathcal{L}\left(X_{n}\right) ; W(X)\right)$ needs to be renormalized at $X_{k}=X \forall k$ only, while at $x_{k}=x$ for all $k, z_{k} \neq z$ for some $k$, it is already defined by the recursion. This fact is responsible for a drastic reduction of renormalization ambiguities in the AdS approach, as compared to renormalization of generalized free fields on Minkowski space.

The renormalization freedom is further reduced by requiring the existence of a boundary limit as a renormalization condition. We shall see in some typical examples (Sect. 3 and Sect. 4) that this condition may require a "field mixing", i.e., perturbative corrections of an interacting Wick monomial by $\mathcal{O}(\kappa)$ times other Wick monomials, in order to cancel perturbative contributions of different scaling dimensions.

We shall show in the next subsection that for $W=: \phi^{l}$ : (no derivatives), the AdS covariant renormalization of $W_{\kappa} \mathcal{L}$ ensures conformal covariance of its boundary limit (2.18)

$$
w_{\kappa \mathcal{L}}(x)=\lim _{z \searrow 0} z^{-\Delta_{\kappa \mathcal{L}}^{W} \cdot W_{\kappa \mathcal{L}}(z, x)}
$$

provided this limit exists, with a suitable (coupling dependent) scale dimension $\Delta_{\kappa \mathcal{L}}^{W}=$ $l \Delta+\mathcal{O}(\kappa)$.

Then we shall illustrate the difference between renormalization on AdS and renormalization on the Minkowski boundary by a flat space model which avoids the technical complications of the curvature.

In Sect. 3, we shall address the renormalizability on AdS and the existence of the boundary limit (2.27) with some case studies.

2.4. Conformal symmetry. In this subsection we assume that for a polynomial interaction $\mathcal{L}(\phi)$, and for $W=: \prod_{j=1}^{l} \partial_{x}^{a_{j}} \phi$ : a Wick polynomial of the free field, an AdSinvariant renormalization of the interacting field $W_{\kappa \mathcal{L}}$ has been achieved, and that the boundary limit (2.18) of $W_{\kappa \mathcal{L}}$ exists with a suitable deformation $l \Delta \mapsto \Delta_{\kappa \mathcal{L}}^{W}$ of the power of $z$ as in (2.19). Under these assumptions we shall prove:

\footnotetext{
3 The "off-shell" formalism in [9] is advantageous only when derivatives of fields appear as arguments of retarded products. In the present study, the field operators may be regarded as "on-shell", i.e., the unperturbed field satisfies the free equation of motion. To simplify the notation, we will, however, write $W_{\kappa} \mathcal{L}=\left(\phi^{l}\right)_{\kappa \phi^{k}}$ when $W=: \phi^{l}:$ and $\mathcal{L}=: \phi^{k}:$.
} 
Proposition 2.1. If the boundary limit (2.18) $w_{\kappa} \mathcal{L}$ of $W_{\kappa \mathcal{L}}$ exists, then it is a scale covariant field with scaling dimension

$$
D_{\kappa \mathcal{L}}^{w}=\sum_{j=1}^{l}\left|a_{j}\right|+\Delta_{\kappa \mathcal{L}}^{W} .
$$

If $W=: \phi^{l}$ : contains no derivatives, then $w_{\kappa} \mathcal{L}$ is a conformally covariant scalar field.

This is, of course, a variant of the central result in $[3,4]$, that the boundary limit of a scalar AdS field, if it exists, automatically inherits unbroken conformal symmetry. The proof given there describes the CFT as a "theory à la Lüscher-Mack" [3,4, Sect. 3] on the cone $C_{2, d}=\left\{\xi \in \mathbb{R}^{d+2}: \xi \cdot \xi=0\right\}$, or a covering thereof. We want to include here a proof that refers directly to the CFT on $d$-dimensional Minkowski spacetime $\mathbb{M}_{d}$, which is (a chart of) the projective cone $\mathrm{PC}_{2, d}=\mathrm{C}_{2, d} /\{\xi \sim \lambda \xi\}$, or a covering thereof. $\left(\mathrm{PC}_{2, d}\right.$ is also known as the Dirac manifold $\mathrm{CM}_{d}$.)

Proof of Prop. 2.1. Let $U$ be the unitary representation of $S O(2, d)$ on the Fock space of the free Klein-Gordon field $\phi$ on AdS, which implements also the conformal transformation of the boundary generalized free field [11]. For the subgroup corresponding to conformal scale transformations on the boundary, we have

$$
\operatorname{Ad} U(\lambda) \phi(z, x) \equiv U(\lambda) \phi(z, x) U(\lambda)^{*}=\phi(\lambda z, \lambda x)
$$

and hence

$$
\operatorname{Ad} U(\lambda) W(X)=\lambda^{\sum_{j}\left|a_{j}\right|} W(\lambda X)
$$

for $W=: \prod_{j=1}^{l} \partial_{x}^{a_{j}} \phi:$. By means of (2.21) and (2.29) we conclude

$$
\operatorname{Ad} U(\lambda) R_{n, 1}\left(\mathcal{L}\left(X_{1}\right), \ldots ; W(X)\right)=\lambda^{\sum_{j}\left|a_{j}\right|} R_{n, 1}(\mathcal{L}(\lambda X), \ldots ; W(\lambda X))
$$

at non-coinciding points (using here that the interaction $\mathcal{L}$ contains no derivatives of $\phi)$. Since we assume that an AdS-invariant renormalization has been achieved, ${ }^{4}$ this identity is maintained in the extension to coinciding points. In terms of the interacting fields (2.20), this gives

$$
\operatorname{Ad} U(\lambda) W_{\kappa \mathcal{L}}(X)=\lambda^{\sum_{j}\left|a_{j}\right|} W_{\kappa \mathcal{L}}(\lambda X)
$$

in the algebraic adiabatic limit. With that and (2.18) we obtain

$$
\begin{aligned}
\operatorname{Ad} U(\lambda) w_{\kappa \mathcal{L}}(x)=\lim _{z \searrow 0} z^{-\Delta_{\kappa \mathcal{L}}^{W} \operatorname{Ad} U(\lambda) W_{\kappa \mathcal{L}}(z, x)} \\
=\lambda^{\sum_{j}\left|a_{j}\right|+\Delta_{\kappa \mathcal{L}}^{W}} \lim _{z \searrow 0}(\lambda z)^{-\Delta_{\kappa \mathcal{L}}^{W}} W_{\kappa \mathcal{L}}(\lambda x, \lambda z)=\lambda^{\sum_{j}\left|a_{j}\right|+\Delta_{\kappa \mathcal{L}}^{W}} w_{\kappa \mathcal{L}}(\lambda x) .
\end{aligned}
$$

This proves the first assertion of the proposition.

We are now going to investigate whether the conclusion (2.33) applies to arbitrary AdS-transformations. Let $t \in S O(2, d):(z, x) \mapsto\left(z^{\prime}, x^{\prime}\right)$ be an AdS-transformation, $\bar{t}$ the conformal transformation induced by $t$ on the boundary, i.e., $\lim _{z \searrow 0} x^{\prime}(z, x)=\bar{t} x$.

\footnotetext{
4 Concrete AdS-invariant renormalization schemes will be presented below.
} 
For free Wick powers $W=: \phi^{l}$ : (without derivatives) and consequently $w=: \varphi^{l}$ : we obtain:

$$
\begin{aligned}
\operatorname{Ad} U(\bar{t}) w(x) & =\lim _{z \searrow 0} z^{-l \Delta} \operatorname{Ad} U(t) W(z, x) \\
& =\lim _{z \searrow 0}\left(\frac{z^{\prime}}{z}\right)^{l \Delta}\left(z^{\prime}\right)^{-l \Delta} W\left(z^{\prime}, x^{\prime}\right)=\lim _{z \searrow 0}\left(\frac{z^{\prime}}{z}\right)^{l \Delta} w(\bar{t} x) .
\end{aligned}
$$

(This argument would fail if $W$ involved derivatives.) Now, AdS-invariance of the volume element $z^{-d-1} d z d^{d} x$ implies

$$
z^{-d-1}=z^{\prime-d-1}\left|\frac{\partial\left(z^{\prime}, x^{\prime}\right)}{\partial(z, x)}\right|
$$

from which it is an easy exercise to conclude that in the limit $z \searrow 0$ (where $\lim _{z \searrow 0} \frac{\partial z^{\prime}}{\partial x}=0$ and $\lim _{z \searrow 0} \frac{\partial z^{\prime}}{\partial z}=\lim _{z \searrow 0} \frac{z^{\prime}}{z}$ ) one obtains

$$
\lim _{z \searrow 0} \frac{z^{\prime}}{z}=\left|\frac{\partial(\bar{t} x)}{\partial x}\right|^{1 / d} .
$$

Thus, the factor in (2.34) equals the conformal prefactor for a covariant field of scaling dimension $l \Delta$.

Turning to interacting fields $W_{\kappa \mathcal{L}}$ for $W=: \phi^{l}:$, the AdS-invariance of the retarded products,

$$
\operatorname{Ad} U(t) R_{n, 1}\left(\mathcal{L}\left(X_{1}\right), \ldots ; W(X)\right)=R_{n, 1}\left(\mathcal{L}\left(t X_{1}\right), \ldots ; W(t X)\right)
$$

for $t \in S O(2, d)$, implies AdS-invariance of the interacting bulk fields in the algebraic adiabatic limit: ${ }^{5}$

$$
\operatorname{Ad} U(t) W_{\kappa \mathcal{L}}(X)=W_{\kappa \mathcal{L}}(t X)
$$

With that we find as before that $w_{\kappa} \mathcal{L}(x)$ is conformally covariant with scaling dimension $\Delta_{\kappa \mathcal{L}}^{W}$ (provided it exists). Namely,

$$
\begin{aligned}
& \operatorname{Ad} U(\bar{t}) w_{\kappa \mathcal{L}}(x)=\lim _{z \searrow 0} z^{-\Delta_{\kappa \mathcal{L}}^{W}} \cdot \operatorname{Ad} U(t) W_{\kappa \mathcal{L}}(z, x) \\
& =\lim _{z \searrow 0}\left(\frac{z^{\prime}}{z}\right)^{\Delta_{\kappa \mathcal{L}}^{W}}\left(z^{\prime}\right)^{-\Delta_{\kappa \mathcal{L}}^{W}} \cdot W_{\kappa \mathcal{L}}\left(z^{\prime}, x^{\prime}\right)=\left|\frac{\partial(\bar{t} x)}{\partial x}\right|^{\Delta_{\kappa \mathcal{L}}^{W} / d} \cdot w_{\kappa \mathcal{L}}(\bar{t} x) .
\end{aligned}
$$

This completes the proof of Prop. 2.1.

\footnotetext{
5 For a special conformal transformation $\bar{t}$ the function $G\left(t^{-1}(z, x)\right)$ does not factorize as (2.17) if $G$ does; but this does not obstruct our procedure thanks to Prop. 8.1 in [6]: in the algebraic adiabatic limit only the constancy of $G$ in the region of interest matters, and this is preserved by the transformation $t$.
} 
2.5. Renormalization on a submanifold: A pedagogical example. We want to illustrate by a simple model that renormalization of a field in $d+1$ dimensions and subsequent restriction to a $d$-dimensional submanifold is not equivalent to renormalization of the restricted fields.

Instead of $\mathrm{CM}_{d}$ as boundary of $\mathrm{AdS}_{d+1}$, we study the 4-dimensional Minkowski space $\mathbb{M}_{4}$ (with coordinates $x=\left(x^{\mu}\right)_{\mu=0, \ldots, 3} \in \mathbb{R}^{4}$ and relative coordinates $y$ ) as a submanifold of the 5-dimensional Minkowski space $\mathbb{M}_{5}$ (with coordinates $X=\left(z \equiv x^{4}, x\right) \in$ $\mathbb{R} \times \mathbb{M}_{4}$ and relative coordinates $\left.Y=(u, y)\right)$. The boundary limit (2.7) corresponds to the restriction to $\mathbb{M}_{4}$ of the fields in $\mathbb{M}_{5}$. The two-point function of a Klein-Gordon field of mass $M \geq 0$ in $\mathbb{M}_{d}$ is given by

$$
\Delta_{M}^{+(d)}(y) \equiv(\Omega, \phi(x+y) \phi(x) \Omega)=\frac{1}{(2 \pi)^{d-1}} \int d^{d} p \theta\left(p^{0}\right) \delta\left(p^{2}-M^{2}\right) e^{-i p y} .
$$

Putting $d=5$ and replacing $y$ by $Y=(u, y)$, this can be viewed as the 2-point function of a generalized free field in $\mathbb{M}_{4}$ with $u$-dependent Källen-Lehmann weight $[3,4]$ :

$$
\Delta_{M}^{+(5)}(Y)=\frac{1}{2 \pi} \int_{M^{2}}^{\infty} d m^{2} \frac{\cos \left(\sqrt{m^{2}-M^{2}} u\right)}{\sqrt{m^{2}-M^{2}}} \Delta_{m}^{+(4)}(y) .
$$

For later reference, we also introduce the corresponding commutator functions

$$
\Delta_{M}^{(d)}(y) \equiv \Delta_{M}^{+(d)}(y)-\Delta_{M}^{+(d)}(-y)
$$

and the retarded propagators

$$
\Delta_{M}^{\mathrm{ret}(d)}(y) \equiv \Delta_{M}^{(d)}(y) \theta\left(y^{0}\right)=\frac{i}{(2 \pi)^{d}} \int d^{d} p \frac{e^{-i p y}}{p^{2}+i p^{0} 0-M^{2}},
$$

such that $\Delta_{M}^{(d)}(y) \theta\left(-y^{0}\right)=-\Delta_{M}^{\operatorname{ret}(d)}(-y)$.

We first investigate the renormalization of the fish diagram (Fig. 1) in $\mathbb{M}_{5}$. This means that we have to extend the distribution

$$
\begin{aligned}
r_{\text {fish }}^{\circ}(Y) & \equiv-i\left(\Omega,\left[: \phi^{2}(X+Y):,: \phi^{2}(X):\right] \Omega\right) \theta\left(-y^{0}\right) \\
& =-2 i\left(\Delta_{M}^{+(5)}(Y)^{2}-\Delta_{M}^{+(5)}(-Y)^{2}\right) \theta\left(-y^{0}\right),
\end{aligned}
$$

which is well defined for $Y \equiv(u, y) \neq 0$ (because $\left[: \phi^{2}(\cdot):,: \phi^{2}(\cdot):\right]$ vanishes for $y^{2}<u^{2}$ ), to a distribution $r_{\text {fish }} \in \mathcal{D}^{\prime}\left(\mathbb{M}_{5}\right)$ (i.e., to $Y=0$ ). The extension has to be such that it does not increase the scaling degree with respect to $Y \rightarrow 0$.

To obtain a solution of the extension problem in $\mathbb{M}_{5}$, we work with the KällenLehmann representation in $\mathbb{M}_{5}$. The square of the 2-point function is given in App. A. Choosing for simplicity the field to be massless, this gives (using (A.1) with $d=5$ and $m_{1}=m_{2}=0$ )

$$
r_{\text {fish }}^{\circ}(Y)=\frac{\left|S_{3}\right|}{8(2 \pi)^{4}} \int_{0}^{\infty} d m^{2} m i \Delta_{m}^{\mathrm{ret}(5)}(-Y) .
$$

The UV divergence of the unrenormalized distribution $r_{\text {fish }}^{\circ}$ shows up in the divergence of the mass integral. The most general $S O(1,4)$ Lorentz invariant extension with the required scaling degree is given by [9]

$$
r_{\text {fish }}^{(\mu)}(Y) \propto\left(-\square_{Y}+\mu^{2}\right) \int d m^{2} \frac{m}{m^{2}+\mu^{2}} i \Delta_{m}^{\operatorname{ret}(5)}(-Y) \quad\left(\mu^{2} \geq 0\right)
$$


depending on a renormalization parameter $\mu$. (The symbol $\propto$ stands for suppressed numerical factors).

We have obtained $r_{\text {fish }}^{(\mu)}$ by renormalizing in $\mathbb{M}_{5}$. We now consider how this distribution would appear when regarded as a distribution on the hypersurface $\mathbb{M}_{4}$ with the transverse difference coordinate $u$ as a parameter. Writing $Y=(u, y)$ and the five-momentum as $(v, p)$, we arrive at

$$
\begin{aligned}
r_{\mathrm{fish}}^{(\mu)}(u, y) & \propto\left(-\square_{Y}+\mu^{2}\right) \int d m^{2} \frac{m}{m^{2}+\mu^{2}} \int d^{4} p e^{i p y} \int d v \frac{e^{-i v u}}{m^{2}+v^{2}-p^{2}-i p^{0} 0} \\
& \propto\left(-\square_{y}+\partial_{u}^{2}+\mu^{2}\right) \int d^{4} p e^{i p y} \int d m^{2} \frac{m}{m^{2}+\mu^{2}} \frac{e^{-|u| \sqrt{m^{2}-p^{2}-i p^{0} 0}}}{\sqrt{m^{2}-p^{2}-i p^{0} 0}} .
\end{aligned}
$$

The appearance of the derivative $\partial_{u}^{2}$ (outside of the integrals) is characteristic for the 5 -dimensional renormalization. One cannot get rid of this operator, because it cannot be shifted under the integral. (The integrand is not differentiable with respect to $u$ at $u=0$ ). It is the reason why 5-dimensional renormalization "as seen from the hypersurface" goes beyond standard 4-dimensional renormalization. One way to understand this fact is that on the hypersurface, the fields $\left.\partial_{z}^{n} \phi(z, x)\right|_{z=0}$ are independent fields which "mix" with $\left.\phi\right|_{z=0}$ upon 5-dimensional renormalization.

In order to exhibit this more clearly, we compare the result of renormalization in the bulk with the alternative procedure of renormalization on the hypersurface, where we have a $z$-dependent family of fields in four dimensions, similar as in (2.6). The label $z$ just distinguishes different generalized free fields $\varphi_{z}(x) \equiv \phi(x, z)$ on the same hypersurface, see [11]. That is, we write the 5-dimensional 2-point functions in the unrenormalized distribution $r_{\text {fish }}^{\circ}$ (2.44) as a $u=z_{1}-z_{2}$-dependent integral over 4-dimensional 2-point functions as in (2.41) (with $M=0$ ), and apply the Källen-Lehmann representation for the resulting products of 2-point functions as in (A.1) with $d=4$. This gives

$$
\begin{aligned}
r_{\text {fish }}^{\circ}(Y)= & -\frac{2 i}{(2 \pi)^{2}} \int_{0}^{\infty} d m_{1}^{2} \int_{0}^{\infty} d m_{2}^{2} \frac{\cos m_{1} u}{m_{1}} \frac{\cos m_{2} u}{m_{2}} \\
& \cdot\left(\Delta_{m_{1}}^{+(4)}(y) \Delta_{m_{2}}^{+(4)}(y)-\Delta_{m_{1}}^{+(4)}(-y) \Delta_{m_{2}}^{+(4)}(-y)\right) \theta\left(-y^{0}\right) \\
= & \int_{0}^{\infty} d m^{2} F\left(m^{2}, u\right) i \Delta_{m}^{\mathrm{ret}(4)}(-y),
\end{aligned}
$$

with

$$
\begin{aligned}
F\left(m^{2}, u\right) \equiv & \frac{2 m^{-2}}{(2 \pi)^{4}} \int_{0}^{\infty} d m_{1} \int_{0}^{\infty} d m_{2} \theta\left(m-m_{1}-m_{2}\right) \\
& \cdot \cos \left(m_{1} u\right) \cos \left(m_{2} u\right) \sqrt{\left(m^{2}-m_{1}^{2}-m_{2}^{2}\right)^{2}-4 m_{1}^{2} m_{2}^{2}}
\end{aligned}
$$

The unrenormalized distribution $r_{\text {fish }}^{\circ}$ exists in $\mathcal{D}^{\prime}\left(\mathbb{M}_{5} \backslash\{0\}\right)$, but for $Y=0$, the mass integral on the right hand side of (2.48) diverges in the region $m^{2} \rightarrow \infty$. Renormalization on $\mathbb{M}_{4}$ means regarding (2.48) as a $u$-dependent Källen-Lehmann representation in $\mathbb{M}_{4}$ and extending it to the diagonal of $\mathbb{M}_{4}$ in an $S O(1,3)$ Lorentz invariant way.

At $u \neq 0$, an extension to $y=0$ is in fact trivial because (2.48) is already defined there, but the extension is non-unique ( $\delta$-functions in $y$ ). In order to extend also to $u=0$ 
( $u=0$ corresponds to two fields on the same hypersurface), one has to consider the most general $S O(1,3)$ Lorentz invariant 4-dimensional renormalization

$$
\tilde{r}_{\text {fish }}^{(\mu)}(Y):=\left(-\square_{y}+\mu^{2}\right) \int d m^{2} \frac{F\left(m^{2}, u\right)}{m^{2}+\mu^{2}} \cdot i \Delta_{m}^{\mathrm{ret}(4)}(-y) \quad\left(\mu^{2} \geq 0\right) .
$$

These distributions exist even in $\mathcal{D}^{\prime}\left(\mathbb{M}_{5}\right)$, have scaling degree $\operatorname{sd}\left(\tilde{r}_{\text {fish }}^{(\mu)}\right)=6=\operatorname{sd}\left(r_{\text {fish }}^{\circ}\right)$ and agree with $r_{\text {fish }}^{\circ}$ for $y \neq 0$. So, $\tilde{r}_{\text {fish }}^{(\mu)}(u, y)$ solves the renormalization (i.e. extension) problem in $\mathbb{M}_{4}$. But it is not a renormalization in $\mathbb{M}_{5}$ because it does not agree with $r_{\text {fish }}^{\circ}$ at $y=0 \wedge u \neq 0$. To see this, we evaluate both (2.48) and (2.50) on a test function $G(Y)=\gamma(u) g(y)$ with $0 \notin \operatorname{supp} \gamma$. Suppressing irrelevant constants, the difference is

$$
\begin{aligned}
\tilde{r}_{\text {fish }}^{(\mu)}(G)-r_{\text {fish }}^{\circ}(G) & \propto \int d m^{2} \int d u \gamma(u) F\left(m^{2}, u\right) \\
& \times \int d^{4} k \hat{g}(k)\left(\frac{k^{2}+\mu^{2}}{\left(m^{2}+\mu^{2}\right)\left(k^{2}-m^{2}\right)}-\frac{1}{k^{2}-m^{2}}\right) \\
& \propto \int d m^{2} \int d u \gamma(u) \frac{F\left(m^{2}, u\right)}{m^{2}+\mu^{2}} \int d^{4} k \hat{g}(k) \\
& \propto g(0) \int \frac{d m^{2}}{m^{2}+\mu^{2}} \int d u \gamma(u) F\left(m^{2}, u\right) .
\end{aligned}
$$

One can actually compute $F\left(m^{2}, u\right)=m^{2} f(m u)$ by using variables $m_{1} u+m_{2} u=m x$ and $m_{1} u-m_{2} u=m y$ in (2.49), giving $f(t) \propto J_{0}(t)+J_{2}(t)=2 t^{-1} J_{1}(t)$. Thus, since $0 \notin \operatorname{supp} \gamma$, the $u$-integral in (2.51) decays $\sim m^{-\frac{1}{2}}$ due to the oscillatory behaviour of $J_{1}$, so that the $m^{2}$-integral is finite as required for a 4-dimensional renormalization. But it obviously does not vanish for generic $\gamma$, as would be required by a 5-dimensional renormalization. This proves the claim. Note that the scale-invariant choice $\mu^{2}=0$ does not alter the conclusion (the mass integral in (2.51) in this case is $\propto \int_{0}^{\infty} J_{1}(t) d t=1$ ). An analogous but more refined argument shows that also when one admits a function $\mu(u)$, the resulting distribution cannot coincide with $r_{\text {fish }}^{\circ}$ for all $(y=0, u \neq 0)$.

The fact that renormalization performed on a submanifold (Eq. (2.50)) does not coincide with proper renormalization in the bulk (Eqs. (2.46), (2.47)), is the main message of this subsection. The breakdown of the bulk symmetry in the hypersurface renormalization is the counterpart of conformal symmetry breaking in AdS-CFT. It can be avoided by bulk renormalization, and subsequent restriction (boundary limit).

\section{Case Studies I: The Interacting Boundary Field $\varphi_{\kappa \phi^{k}}$}

We proceed with some case studies concerning the compatibility of an AdS-invariant renormalization with the existence of the boundary limit. We shall not endeavour the greatest possible generality; e.g., we shall always assume the AdS mass parameter $M^{2}$ to be sufficiently large to avoid the Breitenlohner-Freedman critical behaviour in the range $v^{2} \equiv \frac{d^{2}}{4}+M^{2}<1$ (see, e.g., $\left.[3,4]\right)$.

We start with the perturbative construction of the interacting field $\varphi_{\kappa} \mathcal{L}$ with interaction $\mathcal{L}=: \phi^{k}$ : as a deformation of $\varphi$. The renormalization of $R_{1,1}\left(\mathcal{L}\left(X_{1}\right), \phi(X)\right)$ in this case is unproblematic, but it serves to illustrate the difference between various approaches. In order to work out the boundary limit of the renormalized bulk field $\phi_{\kappa} \mathcal{L}$, 


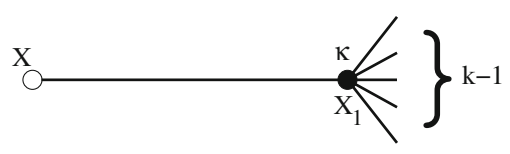

Fig. 2. Factorization of $\phi_{\kappa \mathcal{L}}^{(n)}$

we introduce a general technique of computation (Sect. 3.2) to be used in more general cases as well. In the subsequent section, we shall choose to study the renormalization and boundary limit of the field $\left(\phi^{2}\right)_{\kappa} \mathcal{L}$ because in this case, the perturbative expansion involves a loop diagram (the fish diagram, Fig. 1) already at first order.

Our strategy is to construct the interacting AdS field $\phi_{\kappa \phi^{k}}(X)$, and then take its boundary limit. In the diagrammatic expansion of $\phi_{\kappa \phi^{k}}(X)$, each diagram has a single propagator line extending from $X$ to the first interaction vertex $X_{1}$ (Fig. 2). Therefore, the $z \searrow 0$ behaviour of each diagram is dictated by the same function (apart from potential IR problems), so that the analysis of the limit can be essentially done in the first order. Nontrivial renormalization, in contrast, becomes relevant only at higher order.

To first order perturbation theory $n=1$ we obtain

$$
\phi_{\kappa \phi^{k}}^{(1)}(X)=k \int_{0}^{\infty} \frac{d z_{1}}{z_{1}^{d+1}} \gamma\left(z_{1}\right) \int d^{d} x_{1} g\left(x_{1}\right) \cdot i \Delta_{\mathrm{AdS}}^{\mathrm{ret}}\left(X, X_{1}\right): \phi^{k-1}\left(X_{1}\right): \text {, }
$$

where $\Delta_{\text {AdS }}^{\text {ret }}\left(X, X_{1}\right)=\left(\Delta_{\text {AdS }}^{+}\left(X, X_{1}\right)-\Delta_{\text {AdS }}^{+}\left(X_{1}, X\right)\right) \theta\left(x^{0}-x_{1}^{0}\right)$ is the retarded propagator on $d+1$-dimensional AdS, according to (2.1) given by

$$
\Delta_{\mathrm{AdS}}^{\mathrm{ret}}\left(X, X_{1}\right)=\frac{1}{2}\left(z z_{1}\right)^{d / 2} \int d m^{2} J_{v}(m z) J_{v}\left(m z_{1}\right) \Delta_{m}^{\mathrm{ret}(d)}\left(x-x_{1}\right) .
$$

At this point, one might be tempted to read off the $z \searrow 0$ behaviour directly from (3.2) and the well-known behaviour of the Bessel functions near zero. We shall see, however, that this attempt is too naive, and that the subsequent $z_{1}$-integration in (3.1) changes the limit behaviour substantially.

3.1. Interaction $\mathcal{L}=\kappa \phi$ (field shift). For the trivial case $k=1$ (i.e., the "interaction" amounts just to a shift of the field by a constant), the adiabatic limit $\gamma\left(z_{1}\right)=1, g\left(x_{1}\right)=1$ can be taken directly in (3.1) and yields the expected result

$$
\phi_{\kappa \phi}^{(1)}(X)=\int \frac{d z_{1}}{z_{1}{ }^{d+1}} \int d^{d} x_{1} \cdot i \Delta_{\mathrm{AdS}}^{\mathrm{ret}}\left(X, X_{1}\right)=\frac{1}{M^{2}},
$$

which follows from $\left(\square_{X}+M^{2}\right) i \Delta_{\text {AdS }}^{\text {ret }}\left(X, X_{1}\right)=z^{d+1} \delta\left(z-z_{1}\right) \delta^{d}\left(x-x_{1}\right)$ upon integration over $X_{1}$, using AdS-invariance so that the integral does not depend on $X$. One may also perform the integrations explicitly in the representation (3.2) where the $x_{1}$ integration is obvious from (2.43), and the subsequent $z_{1}$ - and $m$-integrations are carried out using formula (13.24(1)) in [28],

$$
\int_{0}^{\infty} d u u^{\mu} J_{v}(u)=2^{\mu} \frac{\Gamma\left(\frac{1}{2}(1+v+\mu)\right)}{\Gamma\left(\frac{1}{2}(1+v-\mu)\right)} \quad\left(-v-1<\mu<\frac{1}{2}\right) .
$$

Clearly, the shift by a multiple of the "constant field" 1 destroys the existence of the boundary limit with $z^{-\Delta}$. After the subtraction of the vacuum expectation value (i.e., 
undoing the shift), the boundary limit can be taken and reproduces the original boundary field. This trivial example shows that in general, interacting fields of different scaling dimensions may "mix", and the appropriate boundary limits have to be taken after their separation.

3.2. Interaction $\mathcal{L}=\kappa \phi^{2}$ (mass shift). In the case $k=2$, the interaction just amounts to a change of the AdS mass by $\delta M^{2}=-2 \kappa$, so that the perturbed field is just a free field with a different mass. This is an instance of the "Principle of Perturbative Agreement" [19]. Consequently, we expect an anomalous dimension according to $\Delta_{\kappa \phi^{2}}^{\varphi}=$ $d / 2+\sqrt{(d / 2)^{2}+M^{2}-2 \kappa}=\Delta-\kappa / v+\mathcal{O}\left(\kappa^{2}\right)$ to arise. Thus, we are led to study the boundary limit of

$$
\frac{\phi_{\kappa \phi^{2}}(z, x)}{z^{\Delta_{\kappa \phi^{2}}}}=\frac{\phi(z, x)}{z^{\Delta}}+\kappa\left(\frac{\phi_{\kappa \phi^{2}}^{(1)}(z, x)}{z^{\Delta}}+\frac{1}{v} \cdot \frac{\phi(z, x)}{z^{\Delta}} \log z\right)+\mathcal{O}\left(\kappa^{2}\right),
$$

where the first order term (3.1) is

$$
\phi_{\kappa \phi^{2}}^{(1)}(X)=2 \int \frac{d z_{1}}{z_{1}{ }^{d+1}} \gamma\left(z_{1}\right) \int d^{d} x_{1} g\left(x_{1}\right) \cdot i \Delta_{\mathrm{AdS}}^{\mathrm{ret}}\left(X, X_{1}\right) \phi\left(X_{1}\right) .
$$

Indeed, in the partial adiabatic limit $\phi_{\kappa \phi^{2}}^{(1)}$ exhibits a logarithmic $z$-dependence which is precisely cancelled by the combination occurring in (3.5). Namely, (3.6) implies

$$
\left(\square_{X}+M^{2}\right) \phi_{\kappa \phi^{2}}^{(1)}(X)=2 \gamma(z) g(x) \cdot \phi(X),
$$

and consequently, using (2.3),

$$
\left(\square_{X}+M^{2}\right)\left(\phi_{\kappa \phi^{2}}^{(1)}(X)+\frac{\log z}{v} \cdot \phi(X)\right)=\frac{2}{v}\left(\Delta-z \partial_{z}\right) \phi(X)
$$

in the region where $\gamma(z)=1, g(x)=1$. The right-hand side vanishes in the limit $z \searrow 0$ faster than $z^{\Delta}$ because the leading $z^{\Delta}$ behaviour of the unperturbed field is annihilated by the differential operator $\Delta-z \partial_{z}$. Since the Klein-Gordon operator preserves homogeneity in $z$ (except for the $z^{2} \square_{x}$ term which is suppressed at small $z$ ), the combination of fields on the left-hand side also vanishes faster than $z^{\Delta}$, up to a solution of the homogeneous equation. The homogeneous solution can behave $\sim z^{\Delta}$ or $\sim z^{d-\Delta}$.

If we can exclude the latter (dominant) contribution, then it follows that the limit (3.5) at first order in $\kappa$ exists. Unfortunately, the previous argument based on the Klein-Gordon operator cannot discriminate between $\sim z^{\Delta}$ and $\sim z^{d-\Delta}$. We shall therefore develop a more refined analytical method of computation which is "universal" (see Lemma B.1 in App. B) in the sense that it can also be applied when dealing with interactions of higher polynomial degree (Sect. 3.3) and with diagrams with loops (Sect. 4). This method at the same time shows the emergence of the $z^{\Delta} \log z$ terms. The argument is lengthy, with essential parts contained in App. B, but it is crucial for the understanding of the boundary limit.

For the sake of transparency and computational simplicity, we present only the case

$$
d=3 \quad \text { and } \quad M=0 .
$$


The AdS 2-point functions are explicitly known in terms of hypergeometric functions or associated Legendre functions of the second kind [3,4,15]: Let $X=(z, x)$, $X_{1}=\left(z_{1}, x+y\right)\left(z, z_{1} \in \mathbb{R}_{+} ; x, y \in \mathbb{M}^{3}\right)$, and

$$
v=\frac{z^{2}+z_{1}^{2}-y^{2}}{2 z z_{1}} .
$$

$v$ is $\mathrm{AdS}$-invariant. Namely, viewing $\operatorname{AdS}_{d+1}$ as the hypersurface $\xi \cdot \xi=1$ in a $d+2$-dimensional ambient space $\mathbb{R}^{d+2}$ of signature $(+,-\ldots-,+)$, we have

$$
v=\xi \cdot \xi_{1},
$$

hence $v$ is related to the "chordal distance" by $d\left(\xi, \xi_{1}\right)=\left(\xi-\xi_{1}\right)^{2}=2(1-v)$. We expect singularities at $d\left(\xi, \xi_{1}\right)=0(\Leftrightarrow v=1)$ and, due to the identification of $-\xi_{1}$ with $\xi_{1}$, also at $d\left(\xi,-\xi_{1}\right)=0(\Leftrightarrow v=-1)$. Note also that timelike separation between $X$ and $X_{1}$ corresponds to $v \in[-1,1]$. Then for $d=3$,

$$
\Delta_{\mathrm{AdS}}^{+}\left(X_{1}, X\right)=-\frac{1}{4 \pi^{2}} Q_{v-\frac{1}{2}}^{\prime}\left(v+i y^{0} 0\right) \text {. }
$$

Here $Q_{\ell}(u)$ is a solution of Legendre's differential equation

$$
\left(1-u^{2}\right) f^{\prime \prime}-2 u f^{\prime}+\ell(\ell+1) f=0,
$$

which is analytic outside a cut along the real interval $[-1,1]$. For $M=0$, hence $v=\frac{3}{2}$, $\Delta=3$, it is the elementary function

$$
Q_{1}(u)=\frac{u}{2} \log \frac{u+1}{u-1}-1 \Rightarrow Q_{1}^{\prime}(u)=\frac{1}{2}\left(1+u \partial_{u}\right) \log \frac{u+1}{u-1} .
$$

The retarded propagator $\Delta_{\mathrm{AdS}}^{\mathrm{ret}}\left(X, X_{1}\right)=\left(\Delta_{\mathrm{AdS}}^{+}\left(X, X_{1}\right)-\Delta_{\mathrm{AdS}}^{+}\left(X_{1}, X\right)\right) \theta\left(-y^{0}\right)$ is given by the discontinuity across the cut:

$$
\begin{aligned}
4 \pi i \Delta_{\text {AdS }}^{\mathrm{ret}}\left(X, X_{1}\right) & =\left.\frac{1}{2 \pi}\left(1+u \partial_{u}\right) \log \frac{u+1}{u-1}\right|_{u=v-i 0} ^{u=v+i 0} \cdot \theta\left(-y^{0}\right) \\
& =-\left(1+v \partial_{v}\right) \theta(1-|v|) \cdot \theta\left(-y^{0}\right) .
\end{aligned}
$$

This discontinuity is to be understood as a distribution by partial integration w.r.t. $v$ :

$$
H[f]:=-\int d v f(v)\left(1+v \partial_{v}\right) \theta(1-|v|)=\int_{-1}^{+1} d v v \partial_{v} f(v) .
$$

Because we have represented the retarded propagator as a distribution w.r.t. the variable $v$, we have to perform all other integrations (at fixed value of $v$ ) first. We therefore change the integration variables: in spatial polar coordinates, let $y=\left(-t, r \boldsymbol{e}_{\varphi}\right)$, and $w:=y^{2} \equiv t^{2}-r^{2}$. Then the new variables are

$$
v \equiv \frac{z^{2}+z_{1}^{2}-w}{2 z z_{1}}, \quad z_{1}, \quad t \equiv-y^{0}, \quad \varphi .
$$

The measure becomes

$$
d^{3} y \theta\left(-y^{0}\right) \frac{d z_{1}}{z_{1}^{4}} \theta\left(z_{1}\right)=z \cdot d v \cdot \frac{d z_{1}}{z_{1}^{3}} \theta\left(z_{1}\right) \cdot d t \theta(t) \theta\left(t^{2}-w\right) \cdot d \varphi,
$$


where

$$
w=w_{v, z}\left(z_{1}\right)=z^{2}+z_{1}^{2}-2 v \cdot z z_{1} \equiv\left(z_{1} v-z\right)^{2}+\left(1-v^{2}\right) z_{1}^{2} .
$$

There is a dense domain of vectors for which matrix elements $\left(\Psi_{1}, \phi\left(X_{1}\right) \Psi_{2}\right)$ of the distributional field become a smooth function. We then extract the leading $z_{1}$ behaviour and write

$$
\Gamma\left(z_{1}, x^{0}-t, \boldsymbol{x}+\boldsymbol{r} \boldsymbol{e}_{\varphi}\right):=\gamma\left(z_{1}\right) g\left(x_{1}\right) \cdot z_{1}^{-3}\left(\Psi_{1}, \phi\left(z_{1}, x_{1}\right) \Psi_{2}\right) .
$$

This is a smooth function with compact support, because of the cutoff functions $g$ and $\gamma$. At $z_{1}=t=r=0$, it equals the corresponding matrix element of $\varphi(x)$, because $g\left(x_{1}\right)=1$ and $\gamma\left(z_{1}\right)=1$ in the region of interest (partial adiabatic limit). Finally we average over the spatial directions and put

$$
\Gamma_{x}\left(z_{1}, t, r^{2}\right):=\frac{1}{2 \pi} \oint d \varphi \Gamma\left(z_{1}, x^{0}-t, \boldsymbol{x}+\boldsymbol{r} \boldsymbol{e}_{\varphi}\right) .
$$

Then $\Gamma_{x}$ is smooth 6 in all three arguments $\geq 0$, and

$$
\Gamma_{x}(0,0,0)=\left(\Psi_{1}, \varphi(x) \Psi_{2}\right) .
$$

With these preparations, (the matrix element of) the first-order correction (3.6) to the renormalized field becomes

$$
\begin{aligned}
& \left(\Psi_{1}, \phi_{\kappa \phi^{2}}^{(1)}(X) \Psi_{2}\right) \\
& =z \cdot H\left[\left.\int_{0}^{\infty} d z_{1} \int_{0}^{\infty} d t \theta\left(t^{2}-w\right) \cdot \Gamma_{x}\left(z_{1}, t, t^{2}-w\right)\right|_{w=w_{v, z}\left(z_{1}\right)}\right],
\end{aligned}
$$

with the functional $H[\cdot]$ as defined in (3.16). We claim that this equals

$$
\left(\Psi_{1}, \phi_{\kappa \phi^{2}}^{(1)}(X), \Psi_{2}\right)=-\frac{2}{3} z^{3}\left(\log z \cdot \Gamma_{x}(0,0,0)+(\text { regular })\right)
$$

where (regular) stands for a contribution that is regular in $z$ at $z=0$.

The argument goes as follows. For a smooth function $f$ on $\mathbb{R}^{3}$ with compact support, we denote by $I_{0}(v, z)(f)$ the integral

$$
I_{0}(v, z)(f):=\left.\int_{0}^{\infty} d z_{1} \int_{0}^{\infty} d t \theta\left(t^{2}-w\right) f\left(z_{1}, t, t^{2}-w\right)\right|_{w=w_{v, z}\left(z_{1}\right)} .
$$

Thus, to compute (3.23), we have to apply the functional $H$ to $I_{0}(v, z)(f)$ when $f$ equals $\Gamma_{x}$ on $\mathbb{R}_{+}^{3}$.

In App. B, we prove that $I_{0}(v, z)(f)$ is continuous w.r.t. $v$ and differentiable in the range $v^{2}<1$. Thus, the definition (3.16) of $H$ by partial integration is unambiguous, and it is sufficient to know this function at $v^{2}<1$, where $w \geq\left(1-v^{2}\right) z^{2}>0$. In physical terms, this remark means that there are no singular contributions from lightlike $y(w=0)$ : the integration (3.6) can be properly computed by exhausting the backward lightcone "from the inside".

\footnotetext{
${ }^{6}$ It will be important later (App. B) that $\Gamma_{x}$ is regular in the quadratic variable $r^{2}$. This is obvious at $r>0$ because the square root is smooth. At $r=0$, the smoothness can be seen by a Taylor expansion with remainder of $\Gamma\left(z_{1}, x^{0}-t, \boldsymbol{x}+\boldsymbol{r} \boldsymbol{e}_{\varphi}\right)$, because the angular averaging annihilates all odd terms.
} 
In App. B, we also prove that in the range $v^{2}<1, I_{0}(v, z)(f)$ is of the form

$$
\begin{aligned}
& I_{0}(v, z)(f) \\
& \quad=\sum_{0 \leq k \leq \ell \leq 2} A_{k \ell}(f) v^{k} z^{\ell}+z^{2} \cdot \frac{1-v^{2}}{2} \log ((1-v) z) \cdot f(0,0,0)+R_{v, z}(f),
\end{aligned}
$$

where $A_{k \ell}$ are certain distributions that do not depend on $v$ and $z$, while the remainder $R_{v, z}$ is a family of distributions that is differentiable w.r.t. $v$ in the range $v^{2}<1$, and vanishes $\sim z^{3}$ at $z=0$.

Noting that $H\left[v^{0}\right]=H\left[v^{1}\right]=0$, the leading terms are annihilated:

$$
\begin{aligned}
& H\left[I_{0}(v, z)(f)\right]=-\frac{H\left[v^{2}\right]}{2} z^{2} \log z \cdot f(0,0,0) \\
& +z^{2}\left(A_{22}(f)+H\left[\frac{1-v^{2}}{2} \log (1-v)\right] \cdot f(0,0,0)\right)+H\left[R_{v, z}(f)\right] .
\end{aligned}
$$

Thus, with $H\left[v^{2}\right]=\frac{4}{3}$, we have

Proposition 3.1. For any test function $f$ on $\mathbb{R}^{3}$, the limit

$$
\lim _{z \searrow 0} z^{-2}\left\{H\left[I_{0}(v, z)(f)\right]+\frac{2}{3} z^{2} \log z \cdot f(0,0,0)\right\}
$$

is finite.

For $f=\Gamma_{x}$ on $\mathbb{R}_{+}^{3}$, this is our claim (3.24). This ensures that $\phi_{\kappa \phi^{2}}^{(1)}(X)$ decays at least like $z^{3} \log z$, and because of (3.22), it also ensures that $\phi_{\kappa \phi^{2}}^{(1)}(X)+\frac{\log z}{v} \cdot \phi(X)$ (recall $v=\frac{3}{2}$ in (3.5)) decays at least like $z^{\Delta}=z^{3}$. In other words, the boundary limit exists (in first order perturbation theory, and in the obvious weak sense), and is exactly given by the expected correction of the scaling dimension of the boundary field.

Apart from establishing the existence of the (expected) boundary limit, the main message to be drawn from the nontrivial computations in App. B, however, is that

- the origin of the logarithmic term (corresponding to the anomalous dimension) is the range $z_{1}=0$ of the integral (3.6), and not the power law behaviour of the retarded propagator at $z=0$.

3.3. Interactions $\mathcal{L}=\phi^{k}(k>2)$. We now turn to the non-trivial interactions $k>2$. In these cases (3.1) yields

$$
\left(\square_{X}+M^{2}\right) \phi_{\kappa \phi^{k}}^{(1)}(X)=\gamma(z) g(x) \cdot k: \phi^{k-1}(X):,
$$

where the right-hand side $\sim z^{(k-1) \Delta}$ vanishes faster than $z^{\Delta}$. By the same argument as used after (3.8), $\phi_{\kappa \phi^{k}}^{(1)}(X)$ behaves either like $z^{\Delta}$ or like $z^{d-\Delta}$. In the special case $d=3, M=0$, we can explicitly see the absence of the "wrong" contribution $\sim z^{d-\Delta}$, by repeating the explicit computation as in the previous section. Replacing $\phi\left(X_{1}\right)$ by $: \phi\left(X_{1}\right)^{k-1}:$, one gets an additional factor $z_{1}^{3(k-2)}$ in (3.25). Because the logarithmic 
term in this case appears at order $\mathcal{O}\left(z^{3+3(k-2)}\right)$ (Lemma B.1), it is manifest that the first-order term is of order $\mathcal{O}\left(z^{3}\right)$, as desired, and the logarithmic term is suppressed in the limit. Thus, the boundary limit exists without an anomalous dimension.

Although a complete analysis of renormalization at higher-order is beyond the scope of this paper, let us anticipate what happens in the case at hand. First, we observe (see Fig. 2 above) that $\phi_{\kappa \phi^{k}}^{(n)}$ can be written as

$$
\phi_{\kappa \phi^{k}}^{(n)}=k \int_{0}^{\infty} \frac{d z_{1}}{z_{1}^{d+1}} \gamma\left(z_{1}\right) \int d^{d} x_{1} g\left(x_{1}\right) \cdot i \Delta_{\mathrm{AdS}}^{\mathrm{ret}}\left(X, X_{1}\right)\left(\phi^{k-1}\right)_{\kappa \phi^{k}}^{(n-1)}\left(X_{1}\right) .
$$

Thus, in order to renormalize $\phi_{\kappa \phi^{k}}$ at order $n$, one previously has to renormalize $\left(\phi^{k-1}\right)_{\kappa \phi^{k}}$ at order $n-1$. In principle, one has to renormalize "all fields simultaneously", but in practice, for any finite order of any given field it is sufficient to renormalize only a finite number of fields to lower orders.

Thus, assuming recursively that $\left(\phi^{k-1}\right)_{\kappa \phi^{k}}$ has been defined up to $(n-1)^{\text {st }}$ order, and anticipating that its boundary limit exists with an anomalous dimension of order $\mathcal{O}(\kappa)$, then $\left(\phi^{k-1}\right)_{\kappa \phi^{k}}^{(n-1)}$ behaves like $z^{(k-1) \Delta}$ times a polynomial in $\log z$, as $z \searrow 0$. Because the canonical dimension $(k-1) \Delta$ is larger than $\Delta$, the same argument as before applies to ensure that the partial adiabatic limit for $\phi_{\kappa} \phi^{k}$ is unproblematic, and for $z$ sufficiently small (such that $\gamma(z)=1$ ), the equation

$$
\left(\square_{X}+M^{2}\right) \phi_{\kappa \phi^{k}}^{(n)}(X)=k \cdot g(x)\left(\phi^{k-1}\right)_{\kappa \phi^{k}}^{(n-1)}(X)
$$

implies the $z^{\Delta}$ behaviour of $\phi_{\kappa \phi^{k}}^{(n)}(X)$ as $z \searrow 0$. Again, this equation does not yet exclude a term $\sim z^{d-\Delta}$, but an explicit computation as in Lemma B.1 in the special case $d=3$, $M=0$ again shows its absence. We conclude that anomalous dimensions do not arise also in higher orders of perturbation theory.

Actually, one can go beyond this statement: even if the logarithms could be summed (borrowing suitable higher order terms, i.e., violating the proper perturbative systematics) to give rise to an anomalous dimension $\Delta_{\kappa \phi^{k}}^{\phi^{k-1}}$ up to order $n-1$ (see Sect. 4), then the argument would still hold true as long as $\Delta_{\kappa \phi^{k}}^{\phi^{k-1}}>\Delta$ (cf. Lemma B.1 with $\left.n=\Delta_{\kappa \phi^{k}}^{\phi^{k-1}}-\Delta\right)$.

In the next section, we shall discuss the behaviour of "composite fields" $\left(\phi^{2}\right)_{\kappa} \mathcal{L}$. Depending on the interaction, these fields will exhibit finite anomalous dimensions.

3.4. Comparison of bulk vs boundary renormalization schemes. We conclude this section with a comparison of the competing renormalization prescriptions in the case at hand. Concerning the renormalization, we find here significant differences between (a) our procedure, as just outlined, and (b) perturbation theory around the generalized free field $\varphi$ in Minkowski space $\mathbb{M}_{d}$, requiring Poincaré invariance (b1), or in conformal Minkowski space $\mathrm{CM}_{d}$, requiring conformal invariance (b2):

(a) (Renormalization in the bulk) The numerical distribution $r^{\circ}\left(X_{1} ; X\right)=(\Omega$, $\left.R_{1,1}\left(\phi\left(X_{1}\right) ; \phi(X)\right) \Omega\right)$ coincides with the retarded propagator $i \Delta_{\text {AdS }}^{\text {ret }}\left(X, X_{1}\right)$ in AdS. Its extension to the diagonal is uniquely given by (3.2), and there is no freedom of renormalization, because its scaling degree in the relative coordinates equals 
$d-1$ (for $z>0$ ), which is smaller than the dimension of the relative coordinates $(=d+1)[6]$. The boundary behaviour of the resulting fields is dominated by the $z_{1}$-integration near $z_{1}=0$, which depends sensitively on the operator valued distribution with which $r$ is multiplied. It is important to keep in mind that we have renormalized (extended $r^{\circ}$ to the diagonal) first, and then taken the limit $z \searrow 0$ (in the partial adiabatic limit at the boundary).

(b) (Renormalization on the boundary) Doing perturbation theory on the boundary, instead, we have to take the limit $z \searrow 0$ first. This yields the unrenormalized distribution $r_{\varphi}^{\circ}\left(x-x_{1}\right)=\left(\Omega, R_{1,1}\left(\varphi\left(x_{1}\right) ; \varphi(x)\right) \Omega\right)$ :

$$
r_{\varphi}^{\circ}\left(x-x_{1}\right)=i\left[\varphi(x), \varphi\left(x_{1}\right)\right] \theta\left(x^{0}-x_{1}^{0}\right)=\int d m^{2} m^{2 v} i \Delta_{m}^{\mathrm{ret}}\left(x-x_{1}\right) .
$$

This product of distributions exists on $\mathcal{D}\left(\mathbb{M}_{d}\right)$ only in the range $-1<v<0 .{ }^{7}$ For $v \geq 0$ the integral $\int d m^{2} \frac{m^{2 v}}{m^{2}-p^{2}-i p^{0} 0}$ diverges, nevertheless $\left[\varphi(x), \varphi\left(x_{1}\right)\right]$. $\theta\left(x^{0}-x_{1}^{0}\right)$ is well defined for $x \neq x_{1}$, and one is faced with the problem to extend $r_{\varphi}^{\circ}$ from $\mathcal{D}\left(\mathbb{M}_{d} \backslash\{0\}\right)$ to $\mathcal{D}\left(\mathbb{M}_{d}\right)$. One has two options:

- Case (b1). One only requires that the Lorentz invariant extension does not increase the scaling degree (with respect to 0 ) of $r_{\varphi}^{\circ}[6,13]$, which has the value $\operatorname{sd}\left(r_{\varphi}^{\circ}\right)=2 \Delta=d+2 v$. In this case, the retarded propagator is non-unique for $v \geq 0$ : the general solution reads

$$
r_{\varphi}(y)=\left(\mu^{2}-\square_{y}\right)^{[v]+1} \int d m^{2} \frac{m^{2 v} i \Delta_{m}^{\mathrm{ret}}(y)}{\left(\mu^{2}+m^{2}\right)^{[v]+1}}+\sum_{n \leq v} C_{n} \square^{n} \delta(y),
$$

where $\mu>0$ and the $C_{n}$ 's are arbitrary constants (cf. [9, App. C]). Clearly, the renormalization mass $\mu$ and the local terms break the scale invariance (unless $n=v)$.

- Case (b2). Requiring conformal covariance of the extension, a necessary condition is that the homogeneous scaling behaviour of $r_{\varphi}^{\mathrm{o}}$ is maintained: this is an intensification of the requirement in (b1). From (3.33) we see that there is a unique solution for $-1<v \notin \mathbb{N}_{0}$ which is obtained by choosing $\mu=0$ and $C_{n}=0 \forall n$. But if $v \in \mathbb{N}_{0}$, the mass integral is IR-divergent for $\mu=0$, and a scaling covariant retarded propagator does not exist.

\section{Case Studies II: The Interacting Composite Field $\left(\varphi^{2}\right)_{\kappa \phi^{k}}$}

4.1. General considerations. We turn to the field $\left(\phi^{2}\right)_{\kappa} \mathcal{L}$ with interaction $\mathcal{L}=: \phi^{k}$ : $(k \geq 2)$. In this case, there exist three types of diagrams which a priori behave differently as $z \searrow 0$ : those diagrams in which the two interaction vertices connected to the field vertex are distinct and do not belong to a common loop, those in which they are distinct and belong to a common loop, and those in which they coincide (Fig. 3).

Diagrams of the first type factorize into two diagrams as for the field $\phi_{\kappa} \mathcal{L}$ and consequently can be treated as in Sect. 3.3. The second type does not arise in first order.

\footnotetext{
7 The expression on the right side results from the definition of $\varphi(2.8)$. Alternatively, it can be obtained

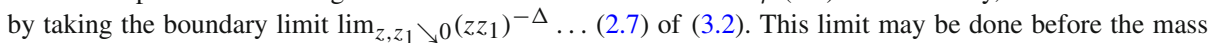
integration in (3.2) iff $-1<v<0$.
} 

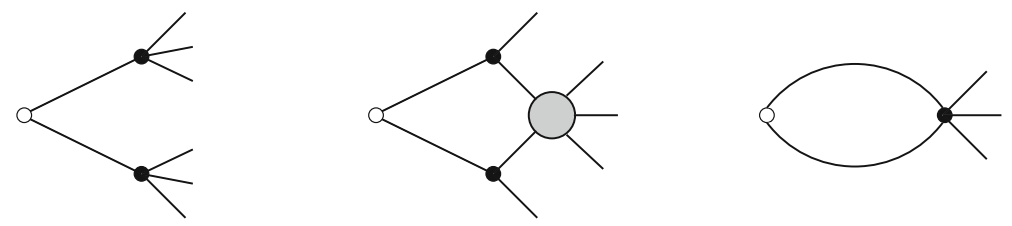

Fig. 3. Three types of diagrams arising in perturbation theory for the interacting field $\left(\phi^{2}\right)_{\kappa} \mathcal{L}(X)$ with interaction $\mathcal{L}=: \phi^{k}$ :

Diagrams of the last type contain the fish diagram (Fig. 1) as a subdiagram, which determines their $z$-dependence. This diagram gives the contribution to $\left(\phi^{2}\right)_{\kappa \phi^{k}}$,

$$
\frac{k(k-1)}{2} \int_{0}^{\infty} \frac{d z_{1}}{z_{1}^{d+1}} \gamma\left(z_{1}\right) \int d x_{1} g\left(x_{1}\right) r_{\mathrm{fish}}\left(X_{1} ; X\right): \phi^{k-2}\left(X_{1}\right): .
$$

In order to define this contribution, the unrenormalized distribution $r_{\text {fish }}^{\circ}\left(X_{1} ; X\right) \equiv$ $\left(\Omega, R_{1,1}^{\circ}\left(\phi^{2}\left(X_{1}\right) ; \phi^{2}(X)\right) \Omega\right)$, given by

$$
r_{\text {fish }}^{\circ}\left(X_{1} ; X\right) \equiv-2 i\left(\Delta_{\text {AdS }}^{+}\left(X_{1}, X\right)^{2}-\Delta_{\text {AdS }}^{+}\left(X, X_{1}\right)^{2}\right) \theta\left(x^{0}-x_{1}^{0}\right)
$$

at $X_{1} \neq X$ (cf. (2.24)), has to be extended to the diagonal $X_{1}=X$. Then we have to study the boundary behaviour $z \searrow 0$ of the renormalized integral (4.1) in the partial adiabatic limit. Our task is to understand the influence of the UV renormalization on the boundary limit.

The unrenormalized distribution (4.2) is real-valued and AdS-invariant. We require that the extension $r_{\text {fish }}\left(X_{1} ; X\right)$ has the same properties:

(I) $r_{\text {fish }}$ is real-valued (i.e., $r_{\text {fish }}(f)^{*}=r_{\text {fish }}\left(f^{*}\right)$ ) and the scaling degree in the relative coordinates $Y=(y, u)$ is not increased by the extension:

$$
\operatorname{sd}_{Y}\left(r_{\text {fish }}(\cdot ; X)\right)=\operatorname{sd}_{Y}\left(r_{\text {fish }}^{\circ}(\cdot ; X)\right)=2 d-2 \quad \forall X .
$$

(II) $r_{\text {fish }}$ is AdS-invariant

$$
r_{\text {fish }}\left(t X_{1} ; t X\right)=r_{\text {fish }}\left(X_{1} ; X\right) \quad \forall t \in S O(2, d) .
$$

In addition, we want to impose the existence of the boundary limit of the interacting field $\left(\phi^{2}\right)_{\kappa \phi^{k}}=: \phi^{2}:+\kappa\left(\phi^{2}\right)_{\kappa \phi^{k}}^{(1)}+\mathcal{O}\left(\kappa^{2}\right)$ as a condition on the renormalization, admitting for an anomalous dimension $2 \Delta+\kappa \delta+\mathcal{O}\left(\kappa^{2}\right)$.

Thus, up to first order of perturbation theory,

$$
\frac{: \phi^{2}(z, x):}{z^{2 \Delta}}+\kappa\left(\frac{\left(\phi^{2}\right)_{\kappa \phi^{k}}^{(1)}(z, x)}{z^{2 \Delta}}-\delta \frac{: \phi^{2}(z, x):}{z^{2 \Delta}} \log z\right)
$$

should converge with $z \searrow 0$. We have already seen that the contributions from the first type of diagrams (Fig. 3) to $\left(\phi^{2}\right)_{\kappa \phi^{k}}^{(1)}$ behave $\sim z^{2 \Delta}$ if $k>2$, and with a logarithmic correction if $k=2$, so that their limit exists separately because $\Delta>0$. Because the only possibly divergent contribution comes from the fish diagram integrated with $: \phi^{k-2}:$, a cancellation against the contribution from an anomalous dimension can occur in (4.5) only if $k=4$ and only if the divergence of $z^{-2 \Delta} r_{\text {fish }}\left(X_{1}, X\right)$ integrated with $: \phi^{2}:$ is logarithmic. Thus, we are led to require 
(III) The renormalized expression (4.1) taken in the partial adiabatic limit and multiplied by $z^{-2 \Delta}$ converges at $z \searrow 0$ if $k \neq 4$, while for $k=4$ it may diverge $\sim \log z \frac{: \phi^{2}(X):}{z^{2 \Delta}}$.

Due to general theorems $[6,17,18]$ there exist extensions which fulfill (I) and (II). For $d \leq 4$, these two requirements reduce the freedom of normalization to

$$
r_{\text {fish }}\left(X_{1} ; X\right)+C z^{d+1} \delta\left(x_{1}-x\right) \delta\left(z_{1}-z\right) .
$$

So there is only one normalization constant $C$ at disposal to fulfill (III). For this reason, we concentrate on $d=3$ and $d=4$ from now on.

Changing the value of $C$ just adds a multiple of : $\phi^{k-2}$ : to $\left(\phi^{2}\right)_{\kappa \phi^{k}}^{(1)}$. If $k=2$ or $k=3$, this term $\sim z^{0}$ or $\sim z^{\Delta}$ must not be present in the boundary limit taken with $z^{-2 \Delta}$, so Condition (III) - if it can be fulfilled - fixes the value of $C$, and thus determines a "field mixing". If $k=4$, the addition just amounts to a multiplicative renormalization of the zero order term. If $k>4$, the addition is ineffective in the boundary limit. In both cases $k \geq 4$, the renormalization parameter $C$ is unconstrained by Condition (III). These a priori conclusions are in perfect agreement with the corresponding conclusions drawn from the analysis of Witten diagrams for correlation functions in the dual approach to the AdS-CFT correspondence [29].

4.2. $d=3, M=0$. Renormalization of the fish diagram on $\mathrm{AdS}_{4}$. The standard strategy [17-19] to renormalize (extend) a distribution like the fish diagram $r_{\text {fish }}^{\circ}$ in curved spacetime is to pass to the scaling limit $\bar{r}_{\text {fish }}^{\circ}$ which gives a distribution in the tangent space at the point $X$. The latter carries the leading UV singularity and can be renormalized as in flat space (with the constant metric $g_{X}$ ), while the less singular "reduced" distribution $r_{\text {fish }}^{\circ \text { red }}=r_{\text {fish }}^{\circ}-\bar{r}_{\text {fish }}^{\circ}$ is (in $d=3$ or $d=4$ ) uniquely extended "by continuity". The problem with this strategy in our situation is that $r_{\text {fish }}^{\text {red }}$ and $\bar{r}_{\text {fish }}$ (the latter being independent of $\Delta$ because the scaling limit loses the information about the AdS mass $M^{2}$ ) behave differently at the boundary, and do not allow us to deduce the boundary behaviour of the integral (4.1).

Let us look more closely at the distribution (4.2). Unfortunately, the AdS KällenLehmann expansion of $\left(\Delta_{\text {AdS }}^{+}\right)^{2}$ is not known explicitly [5], with which one could perform the renormalization in the spirit of (2.46). Instead, we shall use again the explicit form (3.12) of $\Delta_{\mathrm{AdS}}^{+}\left(X_{1}, X\right) \propto Q_{\nu-\frac{1}{2}}^{\prime}$ in $d+1=4$ bulk dimensions, and its elementary expression (3.14) if $M=0$, hence $v=\frac{3}{2}$ and $\Delta=3$.

In order to renormalize (4.2) (i.e., to define the retarded product as a distribution on $\left.\operatorname{AdS}_{4}^{\times 2}\right)$, we adopt the method of differential renormalization [14]: As a distribution on $\mathrm{AdS}_{4}^{\times 2} \backslash\left\{(X, X) \mid X \in \mathrm{AdS}_{4}\right\},(4.2)$ is of the form

$$
r_{\text {fish }}^{\circ}\left(X_{1}, X\right)=j\left(X_{1}, X\right) \theta\left(x^{0}-x_{1}^{0}\right)
$$

with $j\left(X_{1}, X\right) \propto Q_{v-\frac{1}{2}}^{\prime}\left(v-i y^{0} 0\right)^{2}-Q_{v-\frac{1}{2}}^{\prime}\left(v+i y^{0} 0\right)^{2}$. One writes

$$
j\left(X_{1}, X\right)=\square_{X_{1}} J\left(X_{1}, X\right),
$$


where $J$ is an AdS-invariant distribution which vanishes if $X_{1}$ is spacelike separated from $X$, and $\operatorname{sd}(J)<\operatorname{sd}(j)$, so that $J\left(X_{1}, X\right) \theta\left(x^{0}-x_{1}^{0}\right)$ is well-defined as a distribution on $\mathrm{AdS}_{4}^{\times 2}$. One then defines

$$
r_{\text {fish }}\left(X_{1}, X\right):=\square_{X_{1}}\left(J\left(X_{1}, X\right) \theta\left(x^{0}-x_{1}^{0}\right)\right) .
$$

At $X \neq X_{1}$, this differs from the unrenormalized distribution $\theta\left(x^{0}-x_{1}^{0}\right) \cdot \square_{X_{1}} J\left(X_{1}, X\right)$ by a term

$$
\propto \partial_{0}\left(J\left(X_{1}, X\right) \delta\left(x^{0}-x_{1}^{0}\right)\right)+\delta\left(x^{0}-x_{1}^{0}\right) \partial_{0} J\left(X_{1}, X\right) .
$$

The support property of $J$ ensures that this vanishes at $X \neq X_{1}$, hence $r_{\text {fish }}\left(X_{1}, X\right)$ is

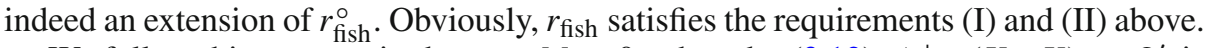

We follow this strategy in the case $M=0$, where by (3.12), $\Delta_{\text {AdS }}^{+}\left(X_{1}, X\right) \sim Q_{1}^{\prime}$ is given explicitly in terms of the elementary function (3.14). We thus obtain

$$
\begin{aligned}
& \left(\Delta_{\text {AdS }}^{+}\left(X_{1}, X\right)\right)^{2} \\
& \quad=\left.\frac{1}{64 \pi^{4}}\left(\left(\log \frac{u+1}{u-1}\right)^{2}+u \partial_{u}\left(\log \frac{u+1}{u-1}\right)^{2}+\left(\frac{u}{u+1}-\frac{u}{u-1}\right)^{2}\right)\right|_{u=v+i y^{0} 0}
\end{aligned}
$$

Here, the first term is a logarithmically bounded function, hence well-defined as a distribution, and consequently also the second. The last term is defined as a distribution by

$$
\left(\frac{1}{v \pm 1+i y^{0} 0}\right)^{2}=-\partial_{v}\left(\frac{1}{v \pm 1+i y^{0} 0}\right) .
$$

We now look for a function $F$ such that

$$
\square_{X_{1}} F(u) \equiv\left(1-u^{2}\right) F^{\prime \prime}(u)-4 u F^{\prime}(u)=Q_{1}^{\prime}(u)^{2}
$$

and

$$
J\left(X_{1}, X\right)=\frac{i}{8 \pi^{4}}\left(F\left(v-i y^{0} 0\right)-F\left(v+i y^{0} 0\right)\right)=0 \quad \text { if } \quad|v|>1 .
$$

Next, we determine the discontinuity along the cut

$$
\delta F(v)=F(v+i 0)-F(v-i 0)
$$

as a distribution. Then, we can define the renormalized fish diagram as

$$
r_{\text {fish }}\left(X_{1}, X\right)=\frac{i}{8 \pi^{4}} \cdot \square_{X_{1}}\left(\delta F(v) \theta\left(-y^{0}\right)\right) .
$$

Proposition 4.1. Equation (4.13) is solved by

$$
\begin{aligned}
F(u)= & \frac{1}{2}\left(\operatorname{Li}_{3} \frac{2}{1-u}+\operatorname{Li}_{3} \frac{2}{1+u}\right)+\frac{1}{6} \frac{d}{d u}\left(\operatorname{Li}_{3} \frac{2}{1-u}-\operatorname{Li}_{3} \frac{2}{1+u}\right) \\
& +\frac{1}{6} \log \frac{u+1}{u-1} \cdot\left(\operatorname{Li}_{2} \frac{2}{1+u}-\operatorname{Li}_{2} \frac{2}{1-u}\right)-\frac{1}{16}\left(\log \frac{u+1}{u-1}\right)^{2} \\
& +\frac{1}{144}\left(u^{2}+3\right) \frac{d}{d u}\left(\log \frac{u+1}{u-1}\right)^{3}+\frac{u}{16} \frac{d}{d u}\left(\log \frac{u+1}{u-1}\right)^{2}+\frac{6-u^{2}}{12\left(u^{2}-1\right)}
\end{aligned}
$$

plus the general solution $C_{1} Q_{1}^{\prime}(u)+C_{2}$ of the homogeneous equation. 
We point out that $F(u)$ is analytic for $u \in \mathbb{C} \backslash[-1,1]$ (see App. C), and that the particular solution given by the proposition is symmetric $(F(-u)=F(u))$, but $Q_{1}^{\prime}(-u)=-Q_{1}^{\prime}(u)$. By writing some terms as derivatives, the boundary values $F(v \pm i \varepsilon)$ are defined as distributions.

Proof. By insertion into (4.13). In App. C we sketch the derivation of (4.17).

Proposition 4.2. The discontinuity $\delta F(v)=F(v+i 0)-F(v-i 0)$ is given by

$$
\delta F(v)=i \pi\left(\theta(1-|v|) h_{0}(v)+\partial_{v}\left(\theta(1-|v|) h_{1}(v)\right)\right),
$$

where

$$
\begin{aligned}
& h_{0}(v)=\frac{1}{3}\left(\operatorname{Li}_{2} \frac{1+v}{2}-\operatorname{Li}_{2} \frac{1-v}{2}\right)+\frac{2}{3} \log \frac{1+v}{1-v}, \\
& h_{1}(v)=\frac{1}{3} \log \frac{1+v}{2} \log \frac{1-v}{2}-\frac{v}{4} \log \frac{1+v}{1-v}+\left(\frac{\pi^{2}}{18}+\frac{5}{12}\right) .
\end{aligned}
$$

Notice that the derivative of $\theta(1-|v|)$ cannot be taken separately, because $h_{1}$ is logarithmically divergent at $v= \pm 1$. Instead, $\delta F$ is understood as a distribution in $v$, where the derivative is defined by partial integration, see below.

Proof. See App. C.

Adding the homogeneous solutions, the second of the integration constants, $C_{2}$, does not contribute to the discontinuity. Thus, the (expected) renormalization freedom consists in adding to (4.16) the term

$$
\begin{aligned}
& \frac{i}{8 \pi^{4}} C_{1} \square_{X_{1}}\left(\left(Q_{1}^{\prime}\left(v-i y^{0} 0\right)-Q_{1}^{\prime}\left(v+i y^{0} 0\right)\right) \theta\left(-y^{0}\right)\right) \\
& \quad=\frac{-i}{2 \pi^{2}} C_{1} \square_{X_{1}} \Delta_{\text {AdS }}^{\mathrm{ret}}\left(X, X_{1}\right)=-\frac{C_{1}}{2 \pi^{2}} z^{4} \delta\left(z_{1}-z\right) \delta^{(3)}\left(x_{1}-x\right) .
\end{aligned}
$$

Remark. (I) In contrast to the renormalization of the massless fish diagram in 4dimensional Minkowski space, the present renormalization on AdS does not require the introduction of a mass scale. This is because there is already a mass scale in the formalism, namely $1 / R^{2}$, where $R$ is the radius of AdS. (In our conventions: $R^{2} \equiv\left(\xi^{0}\right)^{2}-\sum_{k=1,2,3}\left(\xi^{k}\right)^{2}+\left(\xi^{4}\right)^{2}=1$.)

(II) $\delta F$ in Prop. 4.1 is antisymmetric in $v$; however the renormalization freedom (4.20) is symmetric in $v$. Hence, there is a distinguished renormalization: $C_{1}=0$.

The term (4.20) contributes a multiple of : $\phi^{k-2}(X)$ : to the first order term of $\left(\phi^{2}\right)_{\kappa} \phi^{4}$. As discussed in Sect. 4.1, for $k>4$ this terms does not contribute to the boundary limit, while for $k=4$ its boundary limit $: \varphi^{2}(x)$ : exists trivially and amounts to a multiplicative renormalization of $\left(\varphi^{2}\right)_{\kappa} \phi^{4}$. For $k=3$, it produces a "mixing" of the field : $\phi^{2}$ : with $\phi(X)$, and the boundary limit has to be taken of the appropriate mixed field (cf. the end of Sect. 4.3). We shall therefore disregard this term in the sequel.

Thus, (4.16) with $\delta F$ specified by Prop. 4.2 is the starting point for the subsequent analysis of the boundary limit. In that analysis, $\delta F$ is understood as a distribution on the differentiable functions on the interval $(-1,1)$, i.e.,

$$
H_{\mathrm{fish}}[f] \equiv \frac{1}{i \pi} \delta F[f]:=\int_{-1}^{+1} d v\left[h_{0}(v)-h_{1}(v) \partial_{v}\right] f(v) .
$$

The crucial property will be 
Proposition 4.3. The linear functional $H_{\mathrm{fish}}$ vanishes on even powers $f(v)=v^{2 m}$, and

$$
H_{\mathrm{fish}}\left[v^{2 m+1}\right]=\frac{2}{3} \frac{2 m+1}{2 m+2} \sum_{\nu=0}^{2 m+1} J_{v}+\frac{6 m+7}{6} J_{2 m+1}-\frac{5}{6}
$$

where $J_{n}$ are given in (D.6). In particular, $H_{\mathrm{fish}}\left[v^{p}\right]=0$ for $p=0,1,2,3,4$, and $H_{\text {fish }}\left[v^{5}\right]=\frac{4}{81}$.

Proof. The even powers of $v$ are automatically annihilated by $H_{\text {fish }}$ by symmetry under $v \leftrightarrow-v$. For the nontrivial case of the odd powers, see App. D.

4.3. $d=3, M=0$. The boundary limit. Let us first consider the most interesting case of the interaction : $\phi^{4}$ :, i.e., $k=4$. The fish diagram contribution to the first order correction to $\left(\phi^{2}\right)_{\kappa \phi^{4}}$ is given by

$$
\begin{aligned}
& 6 \int d^{3} x_{1} g\left(x_{1}\right) \int_{0}^{\infty} \frac{d z_{1}}{z_{1}^{d+1}} \gamma\left(z_{1}\right) r_{\mathrm{fish}}\left(X_{1}, X\right): \phi^{2}\left(X_{1}\right): \\
& =\frac{6 i}{8 \pi^{4}} \int d^{3} y \int_{0}^{\infty} \frac{d z_{1}}{z_{1}^{d+1}} \delta F(v) \theta\left(-y^{0}\right) \cdot \square_{X_{1}}\left(\gamma\left(z_{1}\right) g(x+y): \phi^{2}\left(z_{1}, x+y\right):\right),
\end{aligned}
$$

where $X_{1}=\left(z_{1}, x_{1}\right), y=x_{1}-x$, and $v=\frac{z^{2}+z_{1}^{2}-y^{2}}{2 z z_{1}}$ as before. To study the boundary limit, we proceed exactly as in Sect. 3.2, when evaluating (3.6). We choose again $d=3$ and $M=0$. Making the same change of variables, we put

$$
\Gamma\left(z_{1}, x^{0}-t, \boldsymbol{x}+r \boldsymbol{e}_{\varphi}\right):=z_{1}^{-6} \square_{X_{1}}\left(\gamma\left(z_{1}\right) g\left(x_{1}\right)\left(\Psi_{1},: \phi^{2}\left(X_{1}\right): \Psi_{2}\right)\right)
$$

and

$$
\Gamma_{x}\left(z_{1}, t, r^{2}\right):=\frac{1}{2 \pi} \oint d \varphi \Gamma\left(z_{1}, x^{0}-t, \boldsymbol{x}+r \boldsymbol{e}_{\varphi}\right) .
$$

Again, $\Gamma_{x}$ is regular at 0 , and

$$
\Gamma_{x}(0,0,0)=-18\left(\Psi_{1},: \varphi^{2}(x): \Psi_{2}\right) .
$$

The factor -18 is produced by the Laplace operator (2.3) when acting on : $\phi\left(z_{1}, x_{1}\right)^{2}: \sim$ $z_{1}^{6}$ at small $z_{1}$. Then we arrive at the matrix element of (4.23),

$$
=-\frac{6 z}{4 \pi^{2}} \cdot H_{\mathrm{fish}}\left[\left.\int_{0}^{\infty} z_{1}^{3} d z_{1} \int_{0}^{\infty} d t \theta\left(t^{2}-w\right) \cdot \Gamma_{x}\left(z_{1}, t, \sqrt{t^{2}-w}\right)\right|_{w=w_{v, z}\left(z_{1}\right)}\right],
$$

which is of the same form as (3.23), except for the additional power $z_{1}^{3}$ (due to the factor $: \phi^{2}$ : in (4.23) as compared to $\phi$ in (3.5)), and with the functional $H$ replaced by $H_{\text {fish }}$ given in (4.21).

The argument in square brackets is of the form $I_{3}(v, z)(f)$ with $f=\Gamma_{x}$ on $\mathbb{R}_{+}^{3}$, as computed in Lemma B.1 of App. B. By the same arguments as before, it is sufficient 
to know it in the range $v^{2}<1$, where it is given by (B.3): there are polynomial terms $\sum_{0 \leq k \leq \ell \leq 5} A_{k \ell}(f) v^{k} z^{\ell}$, a logarithmic contribution

$$
z^{5} \cdot B_{3}(v) \cdot \log ((1-v) z) \cdot f(0,0,0) \quad \text { with } \quad B_{3}(v)=\frac{1}{8} \cdot v\left(1-v^{2}\right)\left(7 v^{2}-3\right),
$$

and a remainder $R_{v, z}(f)=\mathcal{O}\left(z^{6}\right)$ that vanishes in the boundary limit $z \searrow 0$.

By Prop. 4.3, the leading polynomial terms with $k \leq 4$ are annihilated by $H_{\mathrm{fish}}[\cdot]$, so that only the term $H_{\text {fish }}\left[v^{5}\right] A_{55}(f) z^{5}$ survives. The $\log (1-v)$ term in (4.28) produces another constant ${ }^{8}$ times $z^{5} f(0,0,0)$, and the $\log z$-term produces the contribution $-\frac{7}{8} H_{\text {fish }}\left[v^{5}\right] \cdot z^{5} \log z \cdot f(0,0,0)$. Since $H_{\text {fish }}\left[v^{5}\right]=\frac{4}{81}$ (Prop. 4.3), we have thus found the following analog of Prop. 3.1:

Proposition 4.4. For any test function $f$ on $\mathbb{R}^{3}$, the limit

$$
\lim _{z \searrow 0} z^{-5}\left\{H_{\text {fish }}\left[I_{3}(v, z)(f)\right]+\frac{7}{162} z^{5} \log z \cdot f(0,0,0)\right\}
$$

is finite.

Inserting this result with $f=\Gamma_{x}$ and (4.26) into (4.27), we find the first order contribution

$$
(4.23)=-\frac{7 z^{6}}{6 \pi^{2}} \cdot\left(\log z \cdot: \varphi(x)^{2}:+\mathcal{O}(z)\right) .
$$

The absence of all lower order terms establishes the existence of the boundary limit, and the presence of the logarithmic term signals the anomalous dimension of the composite boundary field

$$
\Delta_{\kappa \phi^{2}}^{\phi^{2}}=6-\frac{7}{6 \pi^{2}} \cdot \kappa+\mathcal{O}\left(\kappa^{2}\right)
$$

at first order of perturbation theory.

This establishes the existence of the boundary limit of $\left(\varphi^{2}\right)_{\kappa \phi^{4}}$ in first order perturbation theory, when $M=0$. The result requires the nontrivial cancellations $H_{\mathrm{fish}}[v]=$ $H_{\text {fish }}\left[v^{3}\right]=0$ of Prop. 4.3, involving the precise functions $h_{0}$ and $h_{1}$ of Prop. 4.2 appearing in the renormalized fish diagram. It remains to investigate whether similar cancellations persist for $M \neq 0, d \neq 3$, and at higher orders.

It is now easy to repeat the analysis for the interaction $: \phi^{3}:$, i.e., $: \phi^{2}\left(X_{1}\right): \sim z_{1}^{6}$ on the r.h.s. of (4.23) has to be replaced by $\phi\left(X_{1}\right) \sim z_{1}^{3}$. In this case, the power $z_{1}^{3}$ in the $z_{1}$-integral is absent $(n=0$ in Lemma B.1), hence the logarithmic term $\log z$ arises at order $z^{2}$ with a coefficient $\sim\left(1-v^{2}\right)$. Because $H_{\text {fish }}$ annihilates the quadratic polynomial $B_{0}(v)=\frac{1}{2}\left(1-v^{2}\right)$, but not $B_{0}(v) \log (1-v)$, the first-order diagram will not contain $\log z$ terms, but finite terms $\sim z^{3} \varphi(x)$. This reflects the expected perturbative mixing of the fields $\phi^{2}$ and $\phi$ under the cubic interaction. Accordingly, the boundary limit $z \searrow 0$ should be taken of a suitable combination like $z^{-6}\left(\phi^{2}+\mathcal{O}(\kappa) \phi\right)_{\kappa \phi^{3}}$.

\footnotetext{
8 The factor $\left(1-v^{2}\right)$ in $B_{n}$ in Lemma B.1 ensures the finiteness of $H_{\text {fish }}\left[B_{3}(v) \log (1-v)\right]$.
} 


\section{Conclusion}

We have pursued the strategy of perturbative construction of interacting conformal fields in $d$ dimensions, which proceeds by the perturbative construction of interacting AdS fields in $d+1$ dimensions and subsequently performing a boundary limit. The unperturbed conformal field is a generalized free field (or a Wick product thereof).

This procedure resolves the problematic issues associated with the perturbation theory around generalized free fields, and at the same time drastically reduces the expected infinite arbitrariness involved in its renormalization. The most important benefit is the fact that the boundary fields, if renormalized by this method, do not suffer from the conformal anomaly, i.e., the conformal symmetry is perturbatively preserved.

We find, however, that the existence of the boundary limit is not automatically guaranteed. Requiring its existence may be viewed as another renormalization condition for the AdS field which cannot always be fulfilled. We have pursued a number of case studies involving polynomial interactions of scalar fields. In relevant cases, the boundary limit exists, and the renormalized boundary fields have anomalous dimensions that can be computed. (An anomalous dimension does not mean a conformal anomaly!) Because the exact analytical expressions are quite involved, we have considered only very special cases; but in view of the highly systematic emergence of the cancellations, we believe that the promising results found in these cases pertain also to more general cases.

The method is applicable only when the Lagrangian interaction density of the conformal boundary field is induced by a polynomial interaction on AdS. Such densities are rather special elements of the Borchers class of the generalized free field, which carry a reminiscence of its AdS origin. But in view of the fact that a general perturbation theory for generalized free fields has not yet been formulated, it is encouraging that a successful renormalization can be achieved at least for a limited class of interactions.

There arises an interesting question, concerning the "continuous operator product expansion" for generalized free fields, as discussed in Sect. 2.3. The OPE in the bulk is certainly a discrete sum. Taking the boundary limit, when it exists, should not alter this feature. Recalling that the continuous OPE is caused by the failure of factorization of the weight functions $h\left(k_{1}^{2}, \ldots, k_{l}^{2}\right)$ in (2.13), we are tempted to conjecture the perturbative stability of a discrete OPE for "factorizing" Wick products whenever only the Lagrangian is a non-factorizing generalized Wick product. To establish such a result, one would have to reorganize the OPE of the perturbed limit fields, whose subleading terms are continuous in terms of the unperturbed fields, into a discrete OPE in terms of the perturbed fields.

Acknowledgements. MD profitted from discussions with Günter Scharf and Raymond Stora during an early stage of this work. Extensive discussions with Klaus Fredenhagen clarified many conceptual issues. We thank the anonymous referee for insisting, by his very detailed and qualified inquiries, on more detailed explanations in Sect. 2.3, and for raising the interesting issue of the structure of the OPE.

Open Access This article is distributed under the terms of the Creative Commons Attribution Noncommercial License which permits any noncommercial use, distribution, and reproduction in any medium, provided the original author(s) and source are credited.

\section{A. Källen-Lehmann Representation of $\Delta_{m_{1}}^{+}(y) \Delta_{m_{2}}^{+}(y)$}

Let $\Delta_{m}^{+}(y)$ denote the 2-point function of a massive scalar free field in $d$-dimensional Minkowski space. We are going to prove 


$$
\Delta_{m_{1}}^{+}(y) \Delta_{m_{2}}^{+}(y)=\int_{0}^{\infty} d m^{2} \rho_{m_{1}, m_{2}}\left(m^{2}\right) \Delta_{m}^{+}(y)
$$

with

$$
\begin{aligned}
\rho_{m_{1}, m_{2}}\left(m^{2}\right)= & \frac{\left|S^{d-2}\right|}{4 \cdot 2^{d-3} \cdot(2 \pi)^{d-1}} \\
& \times \theta\left(m-m_{1}-m_{2}\right) \cdot m^{2-d}\left(\left(m^{2}-m_{1}^{2}-m_{2}^{2}\right)^{2}-4 m_{1}^{2} m_{2}^{2}\right)^{\frac{d-3}{2}}
\end{aligned}
$$

with $\left|S^{d}\right|$ the surface of the unit sphere $S^{d}$ in $d+1$ dimensions,

$$
\left|S^{d}\right|=\frac{2 \pi^{\frac{d+1}{2}}}{\Gamma\left(\frac{d+1}{2}\right)} .
$$

From the definitions, and using Lorentz invariance, it is easily seen that the KällenLehmann weight is given by

$\rho_{m_{1}, m_{2}}\left(m^{2}\right)=\frac{1}{(2 \pi)^{d-1}} \int_{V_{+}} d^{d} p_{1} \int_{V_{+}} d^{d} p_{2} \delta\left(p_{1}^{2}-m_{1}^{2}\right) \delta\left(p_{2}^{2}-m_{2}^{2}\right) \delta\left(p_{1}+p_{2}-p\right)$,

where $p \in V_{+}$is any four-momentum such that $p^{2}=m^{2}$. It is convenient to choose $p=(m, \mathbf{0})$ and perform the integrations over the energies $p_{i}^{0}$ first, and evaluate the momentum conservation $\boldsymbol{p}_{2}=-\boldsymbol{p}_{1}$. The resulting integral over $\boldsymbol{p} \equiv \boldsymbol{p}_{1}$ reads in polar coordinates $p=|\boldsymbol{p}|$,

$\rho_{m_{1}, m_{2}}\left(m^{2}\right)=\frac{\left|S^{d-2}\right|}{4(2 \pi)^{d-1}} \int_{0}^{\infty} \frac{d p p^{d-2}}{\sqrt{p^{2}+m_{1}^{2}} \sqrt{p^{2}+m_{2}^{2}}} \delta\left(\sqrt{p^{2}+m_{1}^{2}}+\sqrt{p^{2}+m_{2}^{2}}-m\right)$.

The argument of the $\delta$-function vanishes at

$$
p_{0}=\frac{1}{2 m} \sqrt{\left(m^{2}-m_{1}^{2}-m_{2}^{2}\right)^{2}-4 m_{1}^{2} m_{2}^{2}},
$$

provided $\left(m^{2}-m_{1}^{2}-m_{2}^{2}\right)^{2}-4 m_{1}^{2} m_{2}^{2}>0$ and $m-\left(m_{1}+m_{2}\right)>0$, where the first bound is redundant. From this, we obtain (A.2).

\section{B. The Origin of the Logarithmic Boundary Terms}

We use notations as introduced in Sect. 3.2, with $u \equiv z_{1}$. For a test function $f$ on $\mathbb{R}^{3}$, we denote by $I(u, v, z)$ the integral

$I(u, v, z):=\left.I(w)\right|_{w=w_{v, z}(u)}$, where $I(w):=\int_{0}^{\infty} d t \theta\left(t^{2}-w\right) \cdot f\left(u, t, t^{2}-w\right)$,

and by $I_{n}(v, z)(f)$ the integral

$$
I_{n}(v, z)(f):=\int_{0}^{\infty} u^{n} d u I(u, v, z) \quad(n \geq 0) .
$$

We want to prove: 
Lemma B.1. Let $z>0$. Then $I_{n}(v, z)(f)$ is continuous w.r.t. $v$. In the range $v^{2}<1$, it is of the form

$$
\begin{aligned}
I_{n}(v, z)(f)= & \sum_{0 \leq k \leq \ell \leq n+2} A_{k \ell}(f) v^{k} z^{\ell} \\
& +B_{n}(v) \cdot z^{n+2} \log ((1-v) z) \cdot f(0,0,0)+R_{v, z}(f),
\end{aligned}
$$

if $n \geq 0$ is an integer. Here, $A_{k \ell}$ are distributions, $B_{n}(v)=\frac{1}{2}\left(1-v^{2}\right){ }_{2} F_{1}(-n, n+$ $\left.3 ; 2 ; \frac{1-v}{2}\right)$ is a polynomial of degree $n+2$, and the remainder $R_{v, z}$ is a family of distributions that is differentiable in $v$ in the range $v^{2}<1$, and that vanishes at least $\sim z^{n+3} \log z$ as $z \searrow 0$. If $n=[n]+\varepsilon$ is not an integer, then the first (polynomial) sum extends until $[n]+2$, the logarithmic term is replaced by $C_{n}(v) \cdot z^{[n]+2+\varepsilon} \cdot f(0,0,0)$ with a possibly non-polynomial function $C_{n}$, and the remainder is $\mathcal{O}\left(z^{[n]+3}\right)$.

Remark. The emphasis is here on the various subleading terms after the polynomial terms, because they become the leading ones in different instances of our case studies of the boundary limit, and we expect that this happens also in more general cases. The $\log z$-term is essential for Prop. 3.1 and Prop. 4.4. The $\log (1-v)$-term is used in the last paragraph of Sect. 4.3 , and the $z^{[n]+2+\varepsilon}$-term in the non-integer case is relevant in Sect. 3.3.

Proof. The integrals $I(u, v, z)$ and $I_{n}(v, z)(f)$ are continuous w.r.t. $v$ by definition, because the integrand and the range of integration vary continuously. For the differentiability w.r.t. $v$ when $v^{2}<1$, we note that the dependence on $v$ is only through $w$, and $w=w_{v, z}(u) \geq\left(1-v^{2}\right) u^{2}>0$. Thus $\partial_{v} I(u, v, z)=-2 u z \partial_{w} I(w)$, and

$$
-\partial_{w} I(w)=\frac{1}{2 \sqrt{w}} f(u, \sqrt{w}, 0)+\int_{\sqrt{w}}^{\infty} d t \cdot \partial_{3} f\left(u, t, t^{2}-w\right) .
$$

We now compute the leading derivatives w.r.t. $z$ in the range $v^{2}<1$. Again, the dependence is only through $w=w_{v, z}(u)>0$, and $\partial_{z} I(u, v, z)=-2(u v-z) \partial_{w} I(w)$, hence

$$
\partial_{z}^{\ell} I(u, v, z)=\left.\sum_{k=\left[\frac{\ell+1}{2}\right]}^{\ell} C_{k}^{\ell} \cdot(u v-z)^{2 k-\ell}\left(-\partial_{w}\right)^{k} I(w)\right|_{w=w_{v, z}(u)}
$$

with certain combinatorial coefficients $C_{k}^{\ell}$. Computing $\left(-\partial_{w}\right)^{k} I(w)$, the derivatives can either all go on the integrand, giving

$$
\int_{\sqrt{w}}^{\infty} d t \partial_{3}^{k} f\left(u, t, t^{2}-w\right)
$$

or after $q<k$ derivatives on the integrand, the next derivative goes on the lower boundary, producing $(2 \sqrt{w})^{-1} \partial_{3}^{q} f(u, \sqrt{w}, 0)$, and the remaining $k-q-1$ derivatives produce a sum of terms (neglecting numerical coefficients for the moment)

$$
w^{-k+q+\frac{p}{2}+\frac{1}{2}} \cdot \partial_{2}^{p} \partial_{3}^{q} f(u, \sqrt{w}, 0) \quad \text { with } \quad p+q \leq k-1 \text {. }
$$


Now, at $z=0$, we have $w=u^{2}$, hence the terms (B.6), (B.7) inserted into (B.5) become, respectively,

$$
(u v)^{2 k-\ell} \int_{u}^{\infty} d t \partial_{3}^{k} f\left(u, t, t^{2}-u^{2}\right), \quad(u v)^{2 k-\ell} u^{-2 k+2 q+p+1} \partial_{2}^{p} \partial_{3}^{q} f(u, u, 0) .
$$

To obtain $\partial_{z}^{\ell} I_{n}(v, z)(f)$, these remain to be integrated with $\int_{0}^{\infty} u^{n} d u \ldots$. The $u$-integrals are unproblematic at large $u$ by the falloff of the test function, but they may become singular at $u=0$. The most singular terms are the latter ones in (B.8) when $p=q=0$, i.e., $v^{2 k-\ell} u^{-\ell+1} f(u, u, 0)$. It is then obvious that the $u$-integrals over (B.8) are finite multiples of $v^{2 k-\ell}$, as long as $\ell<n+2$. Thus, for $\ell<n+2,\left.\partial_{z}^{\ell} I_{n}(v, z)(f)\right|_{z=0}$ is finite, and is in fact a polynomial in $v$ of degree $\ell$, because $\left[\frac{\ell+1}{2}\right] \leq k \leq \ell$.

If $n$ is an integer and $\ell=n+2$, the most singular terms $p=q=0$ are

$$
\left.\int_{0}^{\infty} d u u^{n}(u v-z)^{2 k-n-2} w^{-k+\frac{1}{2}} \cdot f(u, \sqrt{w}, 0)\right|_{w=w_{v, z}(u)},
$$

with $\left[\frac{n+3}{2}\right] \leq k \leq n+2$. While all other terms are finite multiples of $v^{2 k-n-2}$ at $z=0$, these terms are logarithmically divergent at $z=0$. To isolate the divergence, we split the integration range into the intervals $(0, U)$ and $(U, \infty)$, for any fixed $U>0$. The latter integral is a finite multiple of $v^{2 k-n-2}$ at $z=0$. In the former, we write $f(u, \sqrt{w}, 0)=f(0,0,0)+(f(u, \sqrt{w}, 0)-f(0,0,0))$, so that the second contribution is also a finite multiple of $v^{2 k-n-2}$ at $z=0$.

The remaining terms, that diverge at $u=0$ when $z=0$, are

$$
f(0,0,0) \cdot \int_{0}^{U} d u u^{n}(u v-z)^{2 k-n-2} w_{v, z}(u)^{-k+\frac{1}{2}}=: I_{n, k}^{\operatorname{div}}(v, z) .
$$

Restoring the suppressed numerical coefficients, these terms sum up to

$$
\sum_{k=\left[\frac{n+3}{2}\right]}^{n+2} C_{k}^{n+2} \frac{1}{2}\left(\frac{1}{2}\right)_{k-1} \cdot I_{n, k}^{\operatorname{div}}(v, z)=-f(0,0,0) \cdot \partial_{z}^{n+2} \int_{0}^{U} d u u^{n} \sqrt{w_{v, z}(u)} .
$$

Equation (B.11) comprises all contributions to $\partial_{z}^{n+2} I_{n}(v, z)(f)$ that are divergent at $z=0$, while all other contributions are polynomials in $v$ of degree $n+2$.

The $u$-integral in (B.11) can be performed explicitly: Introducing the integration variable $s=u-v z$ and the constant $a^{2}:=\left(1-v^{2}\right) z^{2}$, the integrand is a linear combination of terms $s^{m} \sqrt{s^{2}+a^{2}}$. If $m$ is odd, the primitive function is a polynomial in $s, a^{2}$, and $\sqrt{s^{2}+a^{2}}$. Evaluated at the upper and lower values $s=U-v z$ and $s=-v z$, these are regular functions in $v$ and $z$, that possess convergent power series expansions in $v z$ and $z$ in the range $v^{2}<1,0 \leq z<U$. In particular, they contribute further finite values at $z=0$ to (B.11), that are polynomials in $v$ of degree $n+2$.

If $m=2 \mu$ is even, in addition to terms of the previous algebraic type, the primitive functions contain terms of the form

$$
\left.a^{2 \mu+2} \log \left(s+\sqrt{s^{2}+a^{2}}\right)\right|_{s=-v z} ^{U-v z}=\left(z^{2}-v^{2} z^{2}\right)^{\mu+1} \log \frac{U-v z+\sqrt{w_{v, z}(U)}}{-v z+\sqrt{w_{v, z}(0)}} .
$$


The logarithm of the numerator is again a convergent power series as above, and contributes further finite values at $z=0$ to (B.11), that are polynomials in $v$ of degree $n+2$.

But the denominator yields the logarithmic term $\log ((1-v) z)$. Collecting all prefactors, we find the total logarithmic contribution to (B.11) to be given by

$$
(n+2) ! \cdot B_{n}(v) \cdot \log ((1-v) z) \cdot f(0,0,0)
$$

with $B_{n}(v)=\frac{1}{2}\left(1-v^{2}\right) \cdot v^{n}{ }_{2} F_{1}\left(-\frac{n}{2},-\frac{n-1}{2} ; 2 ;-\frac{1-v^{2}}{v^{2}}\right)$. With [1, Eqs. 15.3.19, 15.3.5], this can be brought into the manifestly polynomial form of $B_{n}$ as given in the lemma.

Knowing (the form of) the first $n+2$ derivatives of $I_{n}(v, z)(f)$ at $z=0$, we obtain the claim of the lemma, for $n$ integer.

If $n=[n]+\varepsilon$ is not an integer, then all terms (B.8) give rise to finite integrals $\int u^{n} \ldots$ as long as $\ell \leq[n]+2$, i.e., $\left.\partial_{z}^{\ell} I_{n}(v, z)(f)\right|_{z=0}$ are polynomials in $v$ of degree $\ell$ up to $\ell \leq[n]+2$. However, a scaling argument shows that $\left.\partial_{z}^{[n]+2} I_{n}(v, z)(f)\right|_{z=0}$ has a subleading term of order $O\left(z^{\varepsilon}\right)$ : Namely, the integrands

$$
g_{k}(u, v, z)=u^{n}(u v-z)^{2 k-[n]-2} w_{v, z}(u)^{-k+\frac{1}{2}}
$$

of the leading terms are homogeneous of order $\varepsilon-1$ in $u$ and $z$. Using Euler's equation in the form $\left(z \partial_{z}-\varepsilon\right) g_{k}(u, v, z)=\left(-1-u \partial_{u}\right) g_{k}(u, v, z)=-\partial_{u}\left(u g_{k}(u, v, z)\right)$, this implies

$$
\left(z \partial_{z}-\varepsilon\right) \int_{0}^{U} d u g_{k}(u, v, z)=-U g_{k}(U, v, z),
$$

where $U g_{k}(U, v, 0)=v^{2 k-[n]-2} U^{\varepsilon}$. This differential equation for $\int_{0}^{U} d u g_{k}(u, v, z)$ admits contributions $c_{k}(v) \cdot z^{\varepsilon}$ with undetermined integration constants $c_{k}(v)$, that sum up to $C_{n}(v)$ in the statement of the lemma.

This proves the lemma for non-integer $n$.

The proof of the lemma clearly exhibits the origin of the logarithmic divergence to be the range $z_{1} \approx 0$ of the integration over $z_{1} \equiv u$. Notice also in (B.3) the logarithmic singularity at $v=1$, where $\sqrt{w}=\left|z_{1}-z\right|$. It arises upon integration over $z_{1}$ in the vicinity of $z$, corresponding to the point $X_{1}=X$. This singularity does not lead to divergences, because it is always tamed by the factor $1-v^{2}$ in $B_{n}(v)$.

\section{Details of the Renormalization of the Massless Fish Diagram on AdS}

We work with the convention that the cut of $\log z(z \in \mathbb{C})$ is along $(-\infty, 0]$. As usual we define for $z \in \mathbb{C} \backslash[1, \infty)$,

$$
\operatorname{Li}_{2}(z):=-\int_{C_{z}} d z^{\prime} \frac{\log \left(1-z^{\prime}\right)}{z^{\prime}}, \quad \operatorname{Li}_{3}(z):=\int_{C_{z}} d z^{\prime} \frac{\operatorname{Li}_{2}\left(z^{\prime}\right)}{z^{\prime}},
$$

where $C_{z}$ is any smooth curve from 0 to $z$ which does not intersect $[1, \infty)$. With that $\mathrm{Li}_{2}(z)$ and $\mathrm{Li}_{3}(z)$ are analytic on $\mathbb{C} \backslash[1, \infty)$. Since

$$
\frac{u+1}{u-1} \in(-\infty, 0] \Leftrightarrow u \in[-1,1], \quad \frac{2}{1 \pm u} \in[1, \infty) \Leftrightarrow u \in[-1,1],
$$

the expression (4.17) for $F(u)$ is manifestly analytic for $u \notin[-1,1]$. 
The formula (4.17) for $F(u)$ can be derived by first computing the integral

$$
F^{\prime}(x)=\frac{1}{\left(1-x^{2}\right)^{2}} \int^{x} d t\left(1-t^{2}\right)\left(Q_{1}^{\prime}(t)\right)^{2} \text { for } x \in \mathbb{R},|x|>1,
$$

which gives (after analytic continuation to $z \in \mathbb{C} \backslash[-1,1]$ )

$$
\begin{aligned}
F^{\prime}(z)= & \frac{1}{\left(1-z^{2}\right)^{2}}\left(\frac{2+3 z-z^{3}}{12}\left(\log \frac{z+1}{z-1}\right)^{2}-\frac{z}{3}\right. \\
& \left.+\left(\frac{1}{6}+\frac{z^{2}}{3}\right) \log \frac{z+1}{z-1}+\frac{2}{3} \operatorname{Li}_{2} \frac{2}{1-z}+2 C_{1}\right),
\end{aligned}
$$

where $C_{1}$ is an undetermined constant. A second integration yields $F(u)$ for $u \notin[-1,1]$. Here we use well-known identities for $\mathrm{Li}_{2}$ and $\mathrm{Li}_{3}$ (see, e.g., [21]) and

$$
\begin{aligned}
\frac{1}{z^{2}-1}\left(\log \frac{z+1}{z-1}\right)^{n-1} & =\frac{-1}{2 n} \frac{d}{d z}\left(\log \frac{z+1}{z-1}\right)^{n} \quad(n=2,3), \\
\frac{1}{1 \pm z} \operatorname{Li}_{2} \frac{2}{1 \pm z} & =\mp \frac{d}{d z} \operatorname{Li}_{3} \frac{2}{1 \pm z} .
\end{aligned}
$$

The expressions on the 1.h.s. are problematic, since they have poles at $u= \pm 1$, which overlap with the cut along $[-1,1]$ of the pertinent function in the numerator. But the boundary values at $u=v \pm i 0$ along both sides of the cut of the expressions on the r.h.s. are well defined distributions.

To compute $\delta F(v)=F(v+i 0)-F(v-i 0)$ we use that the complex derivative is given by the infinitesimal differential quotient in any direction, in particular we may choose the direction of the real axis:

$$
\left.\frac{d}{d z} f(z)\right|_{z=v+i w}=\frac{d}{d v} f(v+i w) \text { if } f \text { is holomorphic at } z=v+i w,
$$

and hence

$$
\left.\frac{d}{d z} f(z)\right|_{z=v-i 0} ^{z=v+i 0}=\frac{d}{d v}(f(v+i 0)-f(v-i 0)) .
$$

In addition we give the following formulas:

$$
\begin{aligned}
& \left.\frac{1}{u^{2}-1}\right|_{u=v-i 0} ^{u=v+i 0}=i \pi(\delta(v+1)-\delta(v-1))=i \pi \frac{d}{d v} \theta(1-|v|), \quad v \in \mathbb{R}, \\
& \log \frac{v+i 0+1}{v+i 0-1}=\log \left|\frac{v+1}{v-1}\right|-i \pi \theta(1-|v|), \quad v \in \mathbb{R},
\end{aligned}
$$

$\operatorname{Im} \operatorname{Li}_{2}(x \pm i 0)=-\int_{0}^{x} d t \frac{\operatorname{Im} \log (1-(t \pm i 0))}{t}= \pm \theta(x-1) i \pi \log x, x \in \mathbb{R}$,

$\operatorname{Re}_{L_{2}}(x \pm i 0)=\operatorname{Li}_{2} \frac{x-1}{x}+\frac{1}{2}(\log x)^{2}+\frac{\pi^{2}}{6}-\log x \cdot \log (x-1), x>1$,

$\operatorname{Im~Li}_{3}(x \pm i 0)=\int_{0}^{x} d t \frac{\operatorname{Im~Li}_{2}(t \pm i 0)}{t}= \pm \theta(x-1) \frac{i \pi}{2}(\log x)^{2}, x \in \mathbb{R}$.

With that the result (4.18) is obtained by a straightforward calculation (dropping terms involving $\left.\left(1-v^{2}\right) \cdot \partial_{v} \theta(1-|v|) \equiv 0\right)$. 


\section{Integrals for the Boundary Limit}

Applying the functional $H_{\text {fish }}[f]=\int_{-1}^{+1} d v\left[h_{0}(v)-h_{1}(v) \partial_{v}\right] f(v)$ (with $h_{0}$ and $h_{1}$ as in Prop. 4.2) to odd power functions $f(v)=v^{2 m+1}$, all integrals are of the types

$$
\begin{aligned}
J_{n} & =\int_{-1}^{+1} d v v^{n} \log \frac{1+v}{2}=(-1)^{n} \int_{-1}^{+1} d v v^{n} \log \frac{1-v}{2}, \\
K_{n} & =\int_{-1}^{+1} d v v^{n} \log \frac{1+v}{2} \log \frac{1-v}{2}, \\
L_{n} & =\int_{-1}^{+1} d v v^{n} \operatorname{Li}_{2} \frac{1+v}{2}=(-1)^{n} \int_{-1}^{+1} d v v^{n} \operatorname{Li}_{2} \frac{1-v}{2},
\end{aligned}
$$

so that

$$
H_{\text {fish }}\left[v^{2 m+1}\right]=\frac{2}{3} L_{2 m+1}+\frac{4}{3} J_{2 m+1}-(2 m+1)\left(\frac{1}{3} K_{2 m}-\frac{1}{2} J_{2 m+1}\right)-\frac{\pi^{2}}{9}-\frac{5}{6} .
$$

Since we could not find these integrals in the literature, we sketch their computation here.

In $J_{n}$, we partially integrate $\log \frac{1+v}{2}$ with primitive $(1+v)\left(\log \frac{1+v}{2}-1\right)$. This gives $J_{0}=-2$ and the recursion

$$
J_{n}=-\frac{1+(-1)^{n}}{(n+1)^{2}}-\frac{n}{n+1} J_{n-1},
$$

which is solved by

$$
J_{n}=2 \frac{(-1)^{n+1}}{n+1} \sum_{\nu=0}^{\left[\frac{n}{2}\right]} \frac{1}{2 v+1} .
$$

Summing the geometric series in the integrand of $J_{n}$, we also get

$$
\sum_{n=0}^{\infty} J_{n}=\int_{-1}^{+1} \frac{d v}{1-v} \log \frac{1+v}{2}=\left.\operatorname{Li}_{2} \frac{1-v}{2}\right|_{v=-1} ^{v=+1}=-\frac{\pi^{2}}{6} .
$$

$K_{n}$ vanish if $n$ is odd. Partially integrating $v^{2 m}$ in $K_{2 m}$, expanding $(1-v)^{-1}$ as a geometric series, and using (D.7), we get

$$
K_{2 m}=\frac{2}{2 m+1} \sum_{n=2 m+1}^{\infty} J_{n}=\frac{-2}{2 m+1}\left(\frac{\pi^{2}}{6}+\sum_{n=0}^{2 m} J_{n}\right) .
$$

The integrals $L_{n}$ can be obtained by partial integration of the factor $\operatorname{Li}_{2} \frac{1+v}{2}$ with primitive $(1-v)\left(1-\log \frac{1-v}{2}\right)+(1+v) \operatorname{Li}_{2} \frac{1+v}{2}$, which yields $L_{0}=\frac{\pi^{2}}{3}-2$ and the recursion

$$
(n+1) L_{n}=\frac{\pi^{2}}{3}-(-1)^{n} n\left(J_{n}+J_{n-1}\right)-\frac{1+(-1)^{n}}{n+1}-n L_{n-1}
$$

with solution

$$
(n+1) L_{n}=\frac{\pi^{2}}{6}-(-1)^{n} \sum_{\nu=n+1}^{\infty} J_{v}=\left(1+(-1)^{n}\right) \frac{\pi^{2}}{6}+(-1)^{n} \sum_{\nu=0}^{n} J_{\nu} .
$$

Inserting (D.6), (D.8), (D.10) into (D.4) proves Prop. 4.3. 


\section{References}

1. Abramovitz, M., Stegun, I.A.: Handbook of Mathematical Functions. New York: Dover Publications, 1972

2. Avis, S.J., Isham, C.J., Storey, D.: Quantum field theory in anti-De Sitter space-time. Phys. Rev. D 18, 3565-3576 (1978)

3. Bertola, M., Bros, J., Moschella, U., Schaeffer, R.: A general construction of conformal field theories from scalar anti-de Sitter quantum field theories. Nucl. Phys. B 587, 619-644 (2000)

4. Bertola, M., Bros, J., Gorini, V., Moschella, U., Schaeffer, R.: Decomposing quantum fields on branes. Nucl. Phys. B 581, 575-603 (2000)

5. Bros, J., Epstein, H., Moschella, U.: Towards a general theory of quantized fields on the anti-de Sitter space-time. Commun. Math. Phys. 231, 481-528 (2002)

6. Brunetti, R., Fredenhagen, K.: Microlocal analysis and interacting quantum field theories: renormalization on physical backgrounds. Commun. Math. Phys. 208, 623-661 (2000)

7. Dütsch, M., Fredenhagen, K.: A local (perturbative) construction of observables in gauge theories: the example of QED. Commun. Math. Phys. 203, 71-105 (1999)

8. Dütsch, M., Fredenhagen, K.: Algebraic quantum field theory, perturbation theory, and the loop expansion. Commun. Math. Phys. 219, 5-30 (2001)

9. Dütsch, M., Fredenhagen, K.: Causal perturbation theory in terms of retarded products, and a proof of the Action Ward Identity. Rev. Math. Phys. 16, 1291-1348 (2004)

10. Dütsch, M., Rehren, K.-H.: A comment on the dual field in the AdS-CFT correspondence. Lett. Math. Phys. 62, 171-184 (2002)

11. Dütsch, M., Rehren, K.-H.: Generalized free fields and the AdS-CFT correspondence. Ann. Henri Poincaré 4, 613-635 (2003)

12. Epstein, H.: On the Borchers class of a free field. Nuovo Cim. 27, 886-893 (1963)

13. Epstein, H., Glaser, V.: The role of locality in perturbation theory. Ann. Inst. H. Poincaré A 19, 211-295 (1973)

14. Freedman, D.Z., Johnson, K., Latorre, J.I.: Differential regularization and renormalization: a new method of calculation in quantum field theory. Nucl. Phys. B 371, 353-414 (1992)

15. Fronsdal, C.: Elementary particles in a curved space. II. Phys. Rev. D 10, 589-598 (1974)

16. Haag, R., Kastler, D.: An algebraic approach to quantum field theory. J. Math. Phys. 5, 848-861 (1964)

17. Hollands, S., Wald, R.M.: Local Wick polynomials and time-ordered products of quantum fields in curved spacetime. Commun. Math. Phys. 223, 289-326 (2001)

18. Hollands, S., Wald, R.M.: Existence of local covariant time-ordered products of quantum fields in curved spacetime. Commun. Math. Phys. 231, 309-345 (2002)

19. Hollands, S., Wald, R.M.: Conservation of the stress tensor in perturbative interacting quantum field theory in curved spacetimes. Rev. Math. Phys. 17, 227-312 (2005)

20. Källen, G.: Formal integration of the equations of quantum theory in the Heisenberg representation. Ark. Fysik 2, 371-410 (1950)

21. Lewin, L.: Polylogarithms and Associated Functions. Amsterdam: Elsevier North Holland, 1981

22. Maldacena, J.M.: The large $N$ limit of superconformal field theories and supergravity. Adv. Theor. Math. Phys. 2, 231-252 (1998)

23. Moretti, V.: Comments on the stress-energy tensor operator in curved spacetime. Commun. Math. Phys. 232, 189-221 (2003)

24. Rehren, K.-H.: Algebraic holography. Ann. Henri Poincaré 1, 607-623 (2000)

25. Rehren, K.-H.: Local quantum observables in the AdS-CFT correspondence. Phys. Lett. B 493, 383-388 (2000)

26. Rehren, K.-H.: QFT lectures on AdS-CFT. In: Proceedings of the $3^{\text {rd }}$ Summer School in Modern Mathematical Physics, Zlatibor, Serbia (2004), B. Dragovich (ed.), Belgrade, 2005, pp. 95-118

27. Rühl, W.: Lifting a conformal field theory from $D$-dimensional flat space to $(D+1)$-dimensional AdS space. Nucl. Phys. B705, 437-456 (2005)

28. Watson, G.N.: A Treatise on the Theory of Bessel Functions. Cambridge: Cambridge Univ. Press, 1958 ( $2^{\text {nd }}$ edition)

29. Witten, E.: Anti-de Sitter space and holography. Adv. Theor. Math. Phys. 2, 253-291 (1998)

Communicated by M. Salmhofer 